The AstrophysicAl Journal, 2016, IN PRESS

Preprint typeset using LTEX style emulateapj v. 01/23/15

\title{
FADING CORONAL STRUCTURE AND THE ONSET OF TURBULENCE IN THE YOUNG SOLAR WIND
}

\author{
C. E. DeForest ${ }^{1}$, W. H. Matthaeus ${ }^{2}$, N. M. Viall ${ }^{3}$, And S. R. Cranmer ${ }^{4}$ \\ Draft version October 16, 2018
}

\begin{abstract}
Above the top of the solar corona, the young slow solar wind transitions from low- $\beta$, magnetically structured flow dominated by radial structures, to high- $\beta$, less structured flow dominated by hydrodynamics. This transition, long inferred via theory, is readily apparent in the sky region close to $10^{\circ}$ from the Sun, in processed, background-subtracted solar wind images. We present image sequences collected by the STEREO/HI1 instrument in $2008 \mathrm{Dec}$, covering apparent distances from approximately $4^{\circ}$ to $24^{\circ}$ from the center of the Sun and spanning this transition in large-scale morphology of the wind. We describe the observation and novel techniques to extract evolving image structure from the images, and we use those data and techniques to present and quantify the clear textural shift in the apparent structure of the corona and solar wind in this altitude range. We demonstrate that the change in apparent texture is due both to anomalous fading of the radial striae that characterize the corona, and to anomalous relative brightening of locally dense puffs of solar wind that we term "flocculae;" and show that these phenomena are inconsistent with smooth radial flow, but consistent with onset of hydrodynamic or MHD instabilities leading to a turbulent cascade in the young solar wind.
\end{abstract}

Keywords: Sun: corona - Sun: fundamental parameters - Sun: solar wind - techniques: image processing

\section{INTRODUCTION}

The solar corona is largely structured by the magnetic field of the Sun. At moderate solar altitudes above $2-5 R_{\odot}$, the corona is highly anisotropic, consisting primarily of radial and near-radial structures such as rays (Saito 1965; Newkirk \& Harvey 1968), streamers (Bohlin 1970), pseudostreamers (Wang et al. (2007), and similar dense, open radial structures (Antiochos et al. 2011), all of which may collectively be called "striae" when imaged remotely. The structure is apparent in coronagraphic images of the $\mathrm{K}$ corona (Thomsonscattered light): between about $1^{\circ}$ and $10^{\circ}\left(4 R_{\odot}-40 R_{\odot}\right)$ from the Sun, the appearance of the corona is dominated by radial striae that reflect density differences between the various magnetically structured features. This structuring arises from anisotropic magnetic structure that is easily detected in the mid corona with Thomson scattering (MacQueen et al. 1974), in the EUV corona via collisional line emission (Walker et al. 1988), and even via dispersal of injected particles in the low corona (Raymond et al. 2014). In the mid corona, the magnetic field becomes largely radial (Hundhausen 1972), and variances in flow and density across the magnetic field give rise to the radial striae. In addition to the striae, various localized transient features may be seen: coronal mass ejections (CMEs) and related ejecta (Gosling et al. 1974; McComas et al. 1991), bright "Sheeley blobs" (Sheeley et al. 1997) and related small dense "Viall puffs" that are identifiable in the solar wind (Viall et al. 2010; Viall \& Vourlidas 2015), and myriad small, hard-to-separate density fluctuations that can be identified by motion-filter analysis (DeForest et al. 2014).

The radial striae in coronal structure have been well observed for decades. At some scales they appear to be preserved in the solar wind, as the boundaries between the "fast" solar wind that is associated with coronal holes (Zirker 1977)

\footnotetext{
${ }^{1}$ Southwest Research Institute, 1050 Walnut Street, Boulder, CO, USA

2 University of Delaware

${ }^{3}$ NASA / Goddard Space Flight Center

${ }^{4}$ University of Colorado Boulder
}

and the "slow" solar wind that is associated with the streamer belt and related structures. These striated density structures are often interpreted to be outlining and contrasting magnetic structures, i.e., flux tubes, in the sub-Alfvénic corona. In the corona, the plasma within these flux tubes is fully interactive with regions above and below via magnetohydrodynamic (MHD) waves (Aletti et al. 2000), and in fact MHD waves are routinely observed directly via density fluctuations and Doppler shifts in the low corona (DeForest \& Gurman 1998; Tomczyk \& McIntosh 2009); and adjacent flux tubes can also interact through magnetic reconnection (e.g., Rappazzo et al. 2012). Both of these effects may be viewed as consequences of the anisotropic turbulent MHD relaxation that is expected in the presence of a strong guide field (Montgomery \& Turner 1981; Shebalin et al. 1983), especially at low plasma $\beta$ (Zank $\&$ Matthaeus 1992). These effects preserve structural inhomogeneities introduced by the connectivity of adjacent flux tubes in the mid corona to different portions of the solar surface. In this light, the anisotropic, striated appearance of coronal flux tubes can be seen as a consequence of differing rates of parallel and cross-field relaxation and mixing (Cranmer et al. 2015; Oughton et al. 2015).

In the inner heliosphere just outside the Alfvén surface, where the bulk speed first exceeds the fast mode magnetohydrodynamic (MHD) wave speed, MHD signals cannot propagate back into the corona. However, when sufficiently strong gradients are present, wave signals can still generally overtake lateral expansion and produce local mixing and a local turbulent cascade. Furthermore, with increasing $\beta$ in the outer corona and inner heliosphere the lateral stabilization afforded by the outer corona's radial magnetic field fades to insignificance, enabling isotropization of the fluctuations in velocity and density. ${ }^{5}$ Regions of high shear or high compression in the wind provide a theoretical opportunity for rapid turbulent

\footnotetext{
${ }^{5}$ Even for moderate magnetic field strengths, at sufficiently small scales deep in the inertial range $(\ll 1 \mathrm{Mm})$ it is expected that the anisotropizing effects of the magnetic field persist, because the Alfvén crossing time at length scale $\ell$ decreases as $\sim \ell$ while the nonlinear time scale decreases only as $\sim \ell^{2 / 3}$. The present discussion refers to the outer scale and larger $\ell \gg 1 \mathrm{Mm}$.
} 
evolution over very large scales of multiple $\mathrm{Gm}$ in the superAlfvénic wind due to hydrodynamic instabilities. Stream interaction regions, which begin to develop in the inner heliosphere, are a textbook example of such larger scale interaction (Hundhausen 1972); and long-lived interactions form the familiar corotating interaction regions (Gosling \& Pizzo 1999). High shear regions may be viewed as regions where energy is more strongly injected into fluctuations, harnessing free energy from differential speed of nearby streams. An example of shear-driven turbulence at kinetic scales (Karimabadi et al. 2013) illustrates the complexity of local structure that can be rapidly generated from an initially smooth shear flow. This leads to the supposition that hydrodynamic instabilities associated with differing flow speeds, densities, and wave speeds in adjacent striae must lead to a turbulent solar wind.

Turbulence is, in fact, routinely detected in the slow solar wind at $150 \mathrm{Gm}$ (1 AU), as summarized in a review by Matthaeus \& Velli (2011). The detected turbulence has an average (and highly variable) correlation length of about $1 \mathrm{Gm}$, presumably increasing with distance $r$ as $r^{\alpha}$ with $0.5<\alpha<1$ closer to the Sun (Matthaeus et al. 2005; Breech et al. 2008). Comparisons of wind speed vs. temperature at different altitudes point to ongoing turbulent processing between 0.3 and 4.5 AU (Elliott et al. 2012). Further, the idea that structures smaller than $1 \mathrm{Gm}$ in the solar wind near Earth are at least partially due to turbulent mixing was recently corroborated using imaging of the fluctuating motion of comet tail features (DeForest et al. 2015). Such structures are "small-scale" from the imaging perspective, but are near the boundary between the inertial and energy-containing scale ranges in solar wind turbulence and are therefore "large-scale" from the turbulence theory perspective.

Not all Gm-scale structures in the solar wind arise from turbulence above the corona. Coronal streamers appear to continuously emit trains of small density puffs into the newly formed solar wind, with characteristic radial size scales of a couple of solar radii and time scales of $\sim 90$ minutes (Viall \& Vourlidas 2015); and we refer to these as "Viall puffs." Perevent studies using STEREO/COR and STEREO/HI1 show that these quasi-periodic density structures flow with the slow solar wind, accelerating from $90 \mathrm{~km} \mathrm{~s}^{-1}$ at $2.5 R_{\odot}$ to $285 \mathrm{~km}$ $\mathrm{s}^{-1}$ at $50 R_{\odot}$, and that they expand radially by approximately the same factor in that range (Viall et al. 2010; Viall \& Vourlidas 2015). Viall puffs are too small to follow through the STEREO/HI2 field of view, due to its lower spatial resolution and longer integration times — but they apparently often survive to $1 \mathrm{AU}$, where they are observed by in situ instruments and identified by composition and periodicity (Viall, Kepko \& Spence 2009; Kepko et al. 2016); and they can drive dynamics in Earth's magnetosphere (Kepko, Spence \& Singer 2002; Viall, Spence \& Kasper 2009).

The LASCO C-3 coronagraph (Brueckner et al. 1995) flown on $\mathrm{SOHO}$ (Domingo et al. 1995), produces white-light images out to $7.5^{\circ}\left(30 R_{\odot}\right)$ from the Sun's apparent location. It has been used to detect striae at all position angles, including in the coronal holes, to the limits of its field of view (e.g., DeForest et al. 2001). More recently, the SECCHI instrument suite (Howard et al. 2008) on board the STEREO mission has provided nearly continuous visible light imaging from the inner corona through $90^{\circ}$, via the HI1 and HI-2 instruments (Eyles et al. 2009). These instruments were primarily intended to view CMEs (Harrison et al. 2005) but with the advent of deep-field background subtraction techniques (De-
Forest et al. 2011), they can be used to view both the disposition of the striae at the top of the corona, and the corona's transition to the solar wind at the related locations of the Alfvén surface (at which the wind speed first exceeds the speed of Alfvén or field-aligned fast-mode waves) and the heliospheric $\beta=1$ surface (at which the total gas pressure exceeds the magnetic pressure).

The exact radial locations of the Alfvén surface and the heliospheric $\beta=1$ surface are not well constrained observationally. DeForest et al. (2014) used STEREO/COR2 observations of inbound fluctuations along striae and in the coronal holes as evidence for the Alfvén surface being at least $12 R_{\odot}$ from the Sun over polar coronal holes and $15 R_{\odot}$ in the streamer belt. Models of coronal heating and wind acceleration along open magnetic field lines also make predictions of these locations. One-fluid ZEPHYR models (Cranmer et al. 2007; 2013) reproduced the general latitudinal structure of the coronal wind at solar minimum, as well as in-ecliptic fluctuations associated with quiet Sun regions, and contained a modeled Alfvén surface typically between 7 and $15 R_{\odot}$ and a $\beta=1$ surface typically between 20 and $50 R_{\odot}$. These values are in general agreement with the output of several independent three-dimensional heliospheric simulations (e.g., Lionello et al. 2014; Cohen 2015; Feng et al. 2015), and place these important surfaces somewhere in the lower half of the STEREO/HI1 field of view.

The HI1 field of view is square on the focal plane, and it extends from approximately $3.75^{\circ}$ to $24^{\circ}\left(\sim 15 R_{\odot}\right.$ to $96 R_{\odot}$ ) from the Sun, though that entire range is not available along any one solar-radial chord through the field of view. An important textural transition can be seen in backgroundsubtracted images from that instrument: in the lower/inner part of the field of view, at solar elongation angles near $5^{\circ}$, the radial striae from the streamer belt and related structures dominate the coronal plasma; while in the upper/outer part of the field of view, at elongation angles near $20^{\circ}$, the radial striae no longer dominate and the plasma takes a puffy, or flocculated, appearance. This transition has, to our knowledge, not yet been described in the literature despite nearly a decade of STEREO operations. That is perhaps because of the difficulty of separating the textural change from other observationally important transitions occurring across this field of view. In particular, between $4^{\circ}$ and $20^{\circ}$ solar elongation, the Thomson scattering signal drops by a factor of roughly 125 , falling from roughly $10 \times$ to under $0.1 \times$ the average surface brightness (radiance) of the background starfield. Thus, careful treatment of the background is essential to understanding the shift in appearance.

In the present work, we investigate, characterize, and speculate on the causes of the textural shift in the transition from coronal structure to solar wind structure in the outer reaches of the solar corona, across the HI1 field of view. In Section 2 we describe the data and our methods for preparing them; in Section 3 we present results of visual and quantitative analysis of individual features (3.1) and the statistical ensemble of the images (3.2); in Section 4 we discuss possible explanations for the transition in visual texture from the outer corona to the young solar wind; and in Section 5 we conclude and summarize the results and needed future work.

\section{DATA \& METHODS}

We used synoptic images from the HI1 imager (Eyles et al. 2009) in the SECCHI suite (Howard et al. 2008) aboard the STEREO-A spacecraft, over a single 15 day interval: 2008 
Dec 15 to 2008 Dec 29 inclusive (a period near solar minimum, with well defined streamer belts). The principal concern with imaging wind evolution in this regime is the intrinsic dependence of the solar wind radiance on distance $r$ from the Sun: as the optically thin solar wind propagates outward at roughly constant speed, its density falls as $r^{-2}-$ and its illumination function also falls as $r^{-2}$. These factors combine with the inherent $r$ length scale of of the line of sight, to yield an $r^{-3}$ dependence of apparent surface brightness. That is reflected in an intrinsic variation in the total Thomson scattered radiance of $\varepsilon^{-3}$ at solar elongation angles $\varepsilon$ up to about $30^{\circ}$. The linear relationship between solar distances and elongation angles, at $\varepsilon$ values below $30^{\circ}$, and its breakdown at higher values, have been well described in the literature (e.g., Howard $\&$ Tappin 2009). The radiance of the solar wind thus rapidly drops below the radiance of the background starfield. It is important to note that $r$ is a three-dimensional quantity, and when considering variations along a line of sight, the impact parameter $b$ of a given line of sight corresponds more directly to $\varepsilon$. This is discussed in some detail in the Appendix, and illustrated there in Figure 11.

Fortunately, the HI1 detector response to incident light is quite linear, and the stars are nearly constant brightness. This enables reprocessing of HI1 data to remove the fixed starfield, as described by DeForest et al. (2011). Backgroundsubtracted data produced with their algorithm are available from the STEREO Science Center, as processing levels "L2S" and "L2M". The L2S data are subjected to starfield subtraction using a local-brightness technique that attenuates the background starfield by a factor of 30-100. The L2M data are further processed with a Fourier-domain motion filter, which suppresses the residual starfield by another factor of 10-30 but also suppresses the quasi-stationary radial structures of interest to this study, so we used the slightly noisier L2S data. The effect of L2S processing is illustrated in Figure 1.

We transformed the L2S images to conformal radial coordinates. These are radial coordinates with a logarithmic scale in the radial direction, which (with appropriate scaling of the azimuth and log-radius axes) yields a conformal transformation: one that preserves shape, though not orientation or scale, of small features (e.g., Rudin 1987; DeForest et al. 1997). To avoid aliasing of the starfield and to reduce photon noise by pixel averaging where possible, we resampled the images using optimized spatial filtering keyed to the Jacobian derivative of the transformation (DeForest 2004). The conformal radial coordinate system is particularly useful for two reasons: (1) it enables smoothing across larger larger patches of instrument pixels in the outer reaches, to further beat down background noise where necessary (as demonstrated by DeForest et al. 2001); and (2) it eliminates the visually dominant lateral expansion of the plasma flow across the HI1 field of view, and thereby simplifies visualization of both the usual radial flow and deviations from it.

To further suppress the starfield, we median-filtered each image again, across square patches subtending $0.6^{\circ}$ in solar azimuth in the polar coordinate system. This patch size is equivalent to $21 \times 21 \mathrm{~L} 1$ pixels at the outer edge of the field of view, and $4 \times 4 \mathrm{~L} 1$ pixels at the inner edge.

Next, we removed broad brightness structures via unsharp masking with the minsmooth operator over a circular kernel (DeForest \& Howard 2015). Unsharp masking is the technique of highlighting small scales by subtracting a smoothed version of an image, from the image itself. Minsmooth re- places broad-kernel convolution to produce the smooth "background." It collects a low-percentile value of each pixel's neighborhood, further blurring this neighborhood minimum map with broad-kernel convolution. The result is an effective smooth background for the image, based on feature spatial scale alone. Unsharp masked images made by subtracting a minsmoothed copy of an original are approximately positivedefinite, although they contain only features on spatial scales smaller than the applied neighborhood size. We applied minsmooth unsharp masking on two scales: $14^{\circ}$ diameter to reduce diffuse brightness in the images; and $4.2^{\circ}$ diameter to highlight marker structures in the outer corona and solar wind.

Finally, to suppress residual noise, we averaged across three frames in time. To prevent motion blur from the typical $1^{\circ}$ of outward motion in this interval, we shifted each triplet of frames to their central time, based on a typical radial speed of $350 \mathrm{~km} \mathrm{~s}^{-1}$. This reduced radial motion blur to under $0.2^{\circ}$. We selected this speed by measuring outflow rates of several small features, assuming they were close to the Thomson surface (i.e., that $b \approx r$ for each feature). That assumption is not necessarily warranted for three dimensional studies, but sufficed to identify the bulk motion of the features and reduce motion blur. The measured projected speeds were all within $300-400 \mathrm{~km} \mathrm{~s}^{-1}$, consistent with accepted values for the slow solar wind speed.

Figure 2 shows an example transformed frame and the effect of minsmooth unsharp masking on it. The top panel is a direct copy of the right-hand panel of Figure 1, after local median smoothing to remove the residual starfield. The middle panel shows the effect of a $14^{\circ}$ wide minsmooth unsharp mask, which removes broad diffuse structure without affecting the coronal features. For the remainder of this work, we used these $14^{\circ}$ unsharp-masked images. The bottom panel shows the effect of more aggressive minsmooth unsharp masking, which reveals small-scale features more clearly but also perturbs the image on the spatial scales of interest for our textural study.

The unsharp masked images show radial structure, and its apparent loss, quite strikingly: although the bottom portion of each image is dominated by bright radial structures, and the $\varepsilon^{3}$ scaling should preserve apparent brightness (image pixel value) of wind features as they propagate, the radial structures fade with altitude, nearly vanishing somewhere between $12^{\circ}-18^{\circ}$ from the Sun. In this range, a more nearly isotropic pattern of small puffs comes to dominate, giving the image a flocculated appearance (so called because it resembles the texture of a sheep's side, or that of a flocculated chemical solution, such as ale wort after the cold break; Papazian 2003). The flocculated texture is more apparent in the more aggressively unsharp masked bottom panel of Figure 2, which is included for reference only: all further analysis used the $14^{\circ}$ unsharp masked data.

\section{RESULTS}

The primary result is clearly visible in the bottom two panels of Figure 2: while the radial, striated structure of the coronal streamer belt is obvious and dominates the images at the lower reaches of the HI1 field of view (out to roughly $10^{\circ}$, or $\sim 40 R_{\odot}$ ), that structure largely disappears in the outer half of the field of view. That is surprising, because the $\varepsilon^{3}$ factor in the scaled brightness compensates for both feature expansion and the decrease of the solar illumination function: features evolving only with the average characteristics of the so- 
Level 1 image, crude F subtracted

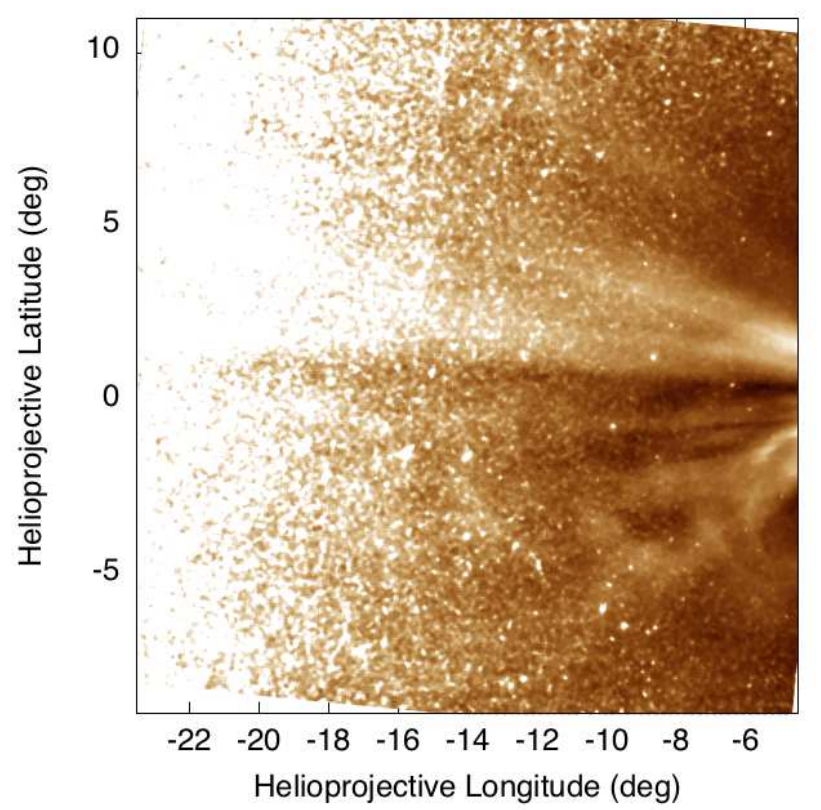

Level 2S image (starfield suppressed)

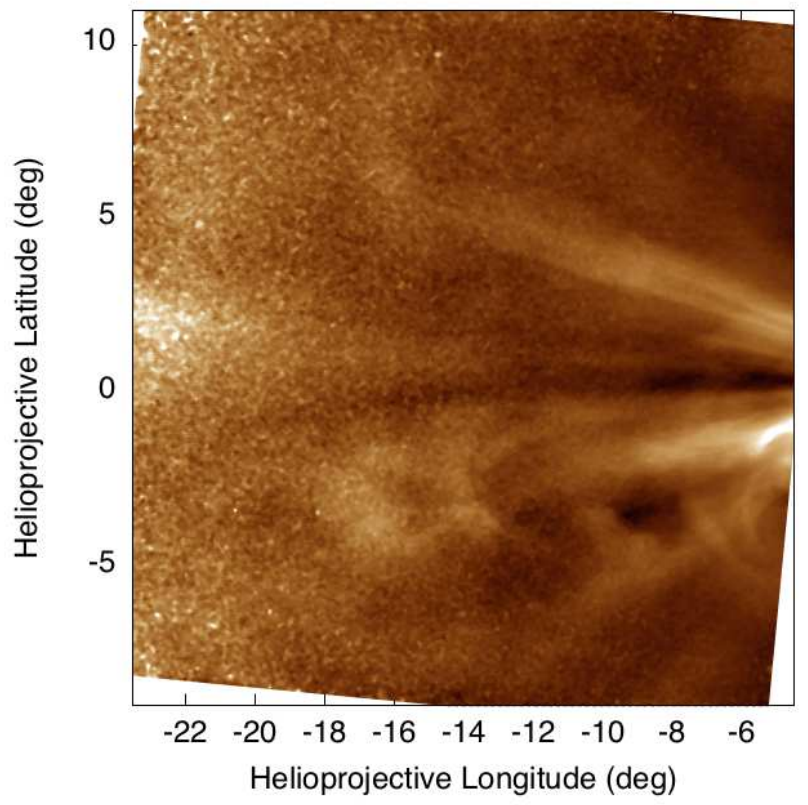

Figure 1. Two copies of the same HI1A image, acquired on 2008 Dec 16 20:09, show how the images are affected by starfield subtraction processing. Both images have been radial-filtered by $\varepsilon^{3}$ scaling, to compensate for the secular falloff in intensity far from the Sun. LEFT: A direct HI1 image, with simple F coronal model subtracted; RIGHT: L2S image suppresses the starfield by a factor of 30-100.

lar wind should maintain the same apparent scaled brightness as they propagate through the scaled images' field of view. We further tested and refined this result in two ways: analysis of individual features and extraction and analysis of an image structure function.

\subsection{Feature Propagation}

Radial features exhibiting the "typical" solar wind behavior of constant-speed flow and conservation of mass are expected to maintain approximately constant brightness in Figure 2. To detail the above introductory discussion of expected brightness scaling, the radiance (surface brightness $B$ ) of a feature is just

$$
B_{\text {feat }}=\pi R_{\odot}^{2} B_{\odot} \sigma_{t} \int\left(n_{e} \frac{1+(\cos \chi)^{2}}{R^{2}}\right) d s,
$$

where $R_{\odot}$ is the solar radius, $B_{\odot}$ is the mean solar radiance, $\sigma_{t}$ is the Thomson scattering cross section, $n_{e}$ is the local electron density, $\chi$ is scattering angle, $R$ is distance from the scattering site to the Sun, and $s$ is distance along a given line of sight (e.g., Howard \& DeForest 2012). From conservation of mass in a constant-radial-flow solar wind, we observe that $n_{e}$ can be written

$$
n_{e}=n_{e 0} R_{0}^{2} R^{-2}
$$

for a constant (against radius) equivalent density at the photosphere $n_{e 0}$ and a reference distance $R_{0}$ (e.g., $\left.1 R_{\odot}\right)$. Thus, Equation 1 simplifies in the constant-flow case to

$$
B_{\text {simple }}=\pi R_{\odot}^{2} B_{\odot} \sigma_{t} n_{e, 0} R_{0}^{2} \int\left(1+(\cos \chi)^{2}\right) R^{-4} d s=k b^{-3},
$$

where $k$ is a constant of proportionality that includes all the terms to the left of the integral and an additional geometrical factor, and $b$ is the impact parameter of the line of sight relative to the Sun. Since the angles under consideration in the HI1 images are less than $30^{\circ}$, the small angle formula applies and the relation $b \approx \varepsilon R_{o b s}$ holds (where $\varepsilon$ is the observed separation angle between an image pixel and Sun center, and $R_{o b s}$ is the distance from the observer to the Sun). Thus, for simple flow,

$$
B_{\text {simple }} \varepsilon^{3} \approx k R_{\text {obs }}^{-3},
$$

which is constant against $\varepsilon$. We tracked several bright features to show that radiance is preserved as expected; two examples are given in Figure 3. The top of row of panels shows a visually striking, compact CME that launched on 2008 Dec 16. The bottom row shows a single bright puff embedded inside a stria. Both features maintain approximately constant brightness in the radially-scaled images.

By contrast, individual radial striae (at locations where there is no particular localized feature to track) fade faster than $\varepsilon^{-3}$. The right hand side of Figure 2 shows this effect in broad context: although the figure uses scaled brightness, the striae visibly fade before reaching the outer limit of the field of view. Figure 4 shows two particular examples of fading radial striae, extracted as in Figure 3 to follow evolution of a particular patch of solar wind as it propagates outward.

Qualitatively, the striae (vertical stripes) in Figure 4 fade continuously from bottom to top in these tracked sequences, dropping below the noise floor. To quantify this fading, and verify that it is not an artifact of the rising noise floor, we smoothed the final images in the vertical direction to bring down the noise floor still further and identify whether the radial stria is still present. To verify whether any identified structure was due to the smoothing direction, we also carried out the identical smoothing operation in the horizontal direction. The results are in Figure 5, which shows clear remnant 

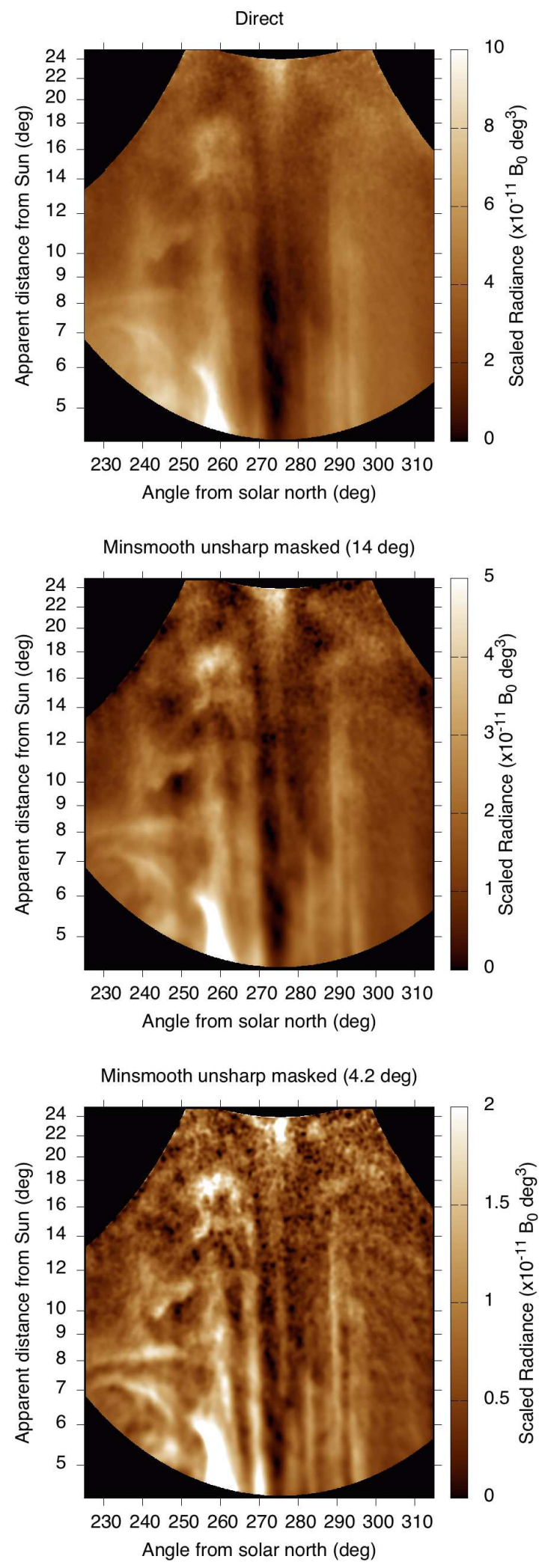

Figure 2. Radial structure is visible in conformal radial coordinates. TOP: the right frame from Fig. 1, after further median filtering, shows fading radial structure. MIDDLE: minsmooth unsharp masking with a $14^{\circ}$ wide kernel hides diffuse brightness, revealing structure. BOTTOM: minsmooth unsharp masking with a $4.2^{\circ}$ wide kernel highlights both radial structure and transient ejecta. See also the accompanying movie in the digital version of this article, which depicts the full data set as processed in the middle panel. For further analysis we used frames similar to the middle panel. vertical structure at the smoothed noise floor after smoothing across $1.5^{\circ}$ of radial extent or the equivalent $\left(6^{\circ}\right)$ in azimuthal extent (all at $20^{\circ}$ elongation).

Although (just) visible in the smoothed images, the remnant radial structure is attenuated by a factor of $3-5$ at $20^{\circ}$ elongation, compared to its scaled brightness at $6^{\circ}$. Note that the important parameter in Figure 5 is the peak-to-peak amplitude of the visible striations, not their absolute brightness: the necessary background subtraction and minsmooth unsharp masking steps invalidate the zero-point measurement.

The fading of the radial striae indicates a real attenuation of their emission compared to a radially propagating, massconserving, solar wind whose speed is independent of radius. The result is strong: by starting with the flat-fielded Level 1 data, avoiding any motion filtering beyond simple starfield removal, performing a control analysis by tracking compact features, and demonstrating persistence of the features despite anisotropic smoothing, we have eliminated most credible causes of potential confusion or loss-of-signal artifacts.

In parallel with the radial fading of the long, anisotropic radial striae in the corona, smaller bright features become more prominent, giving the plasma a puffy or flocculated appearance. The Sheeley et al. (1997) blobs and the Viall et al. (2010) puffs arise from the low corona and do not fade in scaled brightness as they propagate, becoming part of the flocculated gestalt. In addition, there are small puffs with similar size scales that fade in from invisibility in the moving frame of reference of the wind itself, and are therefore visible primarily in the outer field of view. We refer to these brightening puffs as "flocculae." Figure 6 illustrates two of them. The flocculae are in general fainter and larger than the puffs visible in the lower portion of the field of view, appear to grow in scaled brightness as they propagate, and can best be detected only outside of $\sim 10^{\circ}$, fading in even as the striae fade out with altitude.

It is this combination of the fading striae and the growing flocculae that gives rise to the flocculated appearance of the outer HI1 field of view in properly background-subtracted image sequences. In particular, if the HI1 image quality were degrading sufficiently to obscure the striae, the flocculae and other localized features would be equally obscured. The fact that particular classes of feature, propagating with the solar wind, grow in amplitude, even as others fade, reveals that simple loss of contrast or related image degradation is not causing the change in image texture across the field of view: the textural shift reflects a shift in the spatial form of density fluctuations across this field of view.

\subsection{Structure Function Analysis}

In addition to anecdotal analysis of particular striations in the corona, we characterized the evolution of image structure across the HI1 field of view using structure functions (ShulzDuBois \& Rehberg 1981; Cover \& Thomas 1991), which summarize the variability of a data set at different scales and directions. Given a three-dimensional dataset such as our HI1 image sequence, $h(x, y, t)$, the second-order structure function is a six-dimensional functional in both the independent variables and their offsets:

$S_{x y t}(x, y, t, \Delta x, \Delta y, \Delta t) \equiv(h(x, y, t)-h(x-\Delta x, y-\Delta y, t-\Delta t))^{2}$.

$S_{x y t}$ is useful because it can be averaged across neighborhoods of the primary variables' domains, to yield a locationdependent measure of structure vs. scale in the datasets. We 


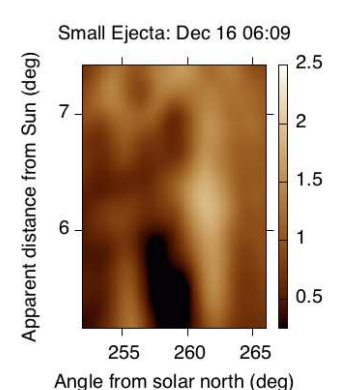

Angle from solar north (deg)

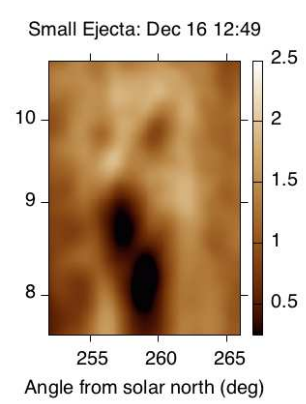

Puff: Dec 23 13:29

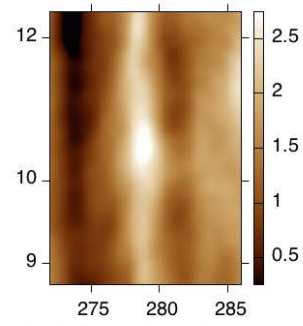

Angle from solar north (deg)

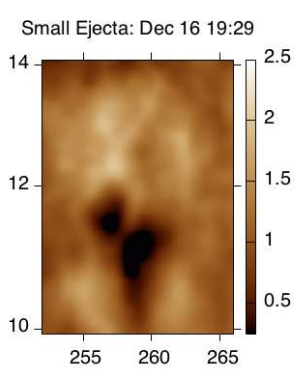

Angle from solar north (deg)

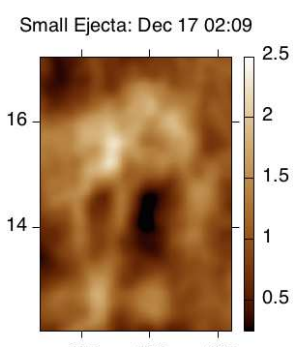

$255 \quad 260 \quad 265$ Angle from solar north (deg)

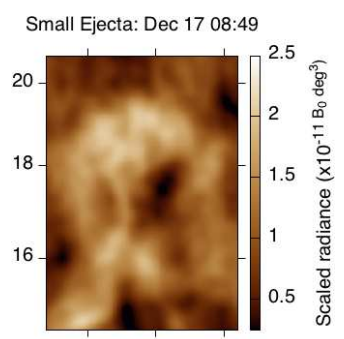

$\begin{array}{lll}255 & 260 \quad 265\end{array}$ Angle from solar north (deg)

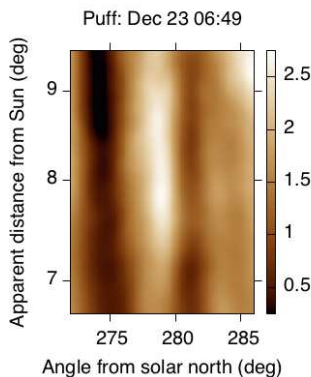

Puff: Dec 23 20:09

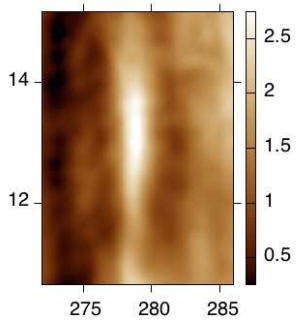

Angle from solar north (deg)

Puff: Dec 24 02:49

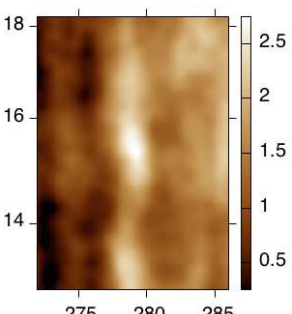

Angle from solar north (deg)
Puff: Dec 24 09:29

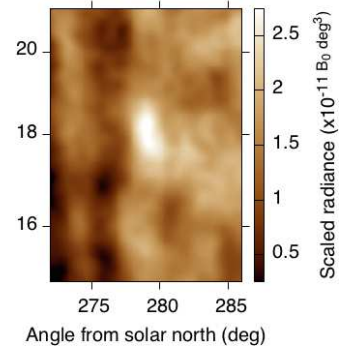

Figure 3. Tracked evolution sequences of two typical bright features (identified inside of $10^{\circ}$ from the Sun) reveal that Equation 4 holds: both features maintain approximately constant brightness in the radially-scaled images, as can be seen by reference to the color bars at right of each panel. They also exhibit the well known "pancake effect" from radial propagation; this appears as radial squashing in the conformal polar coordinates. See also the corresponding movies in the digital version of this article. TOP: a mini-CME. BOTTOM: a localized, dense puff within a stria.
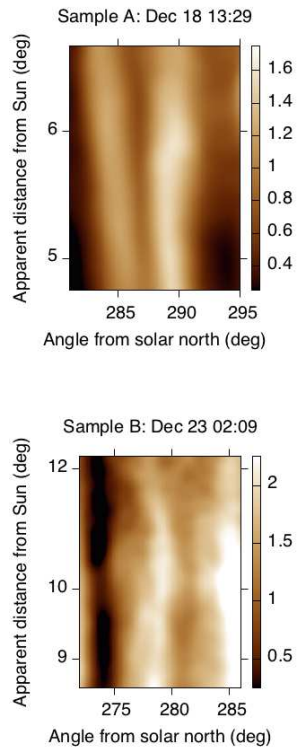

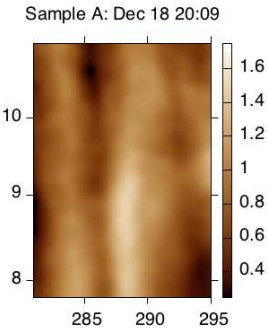

Angle from solar north (deg)

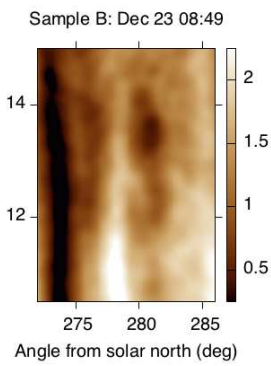

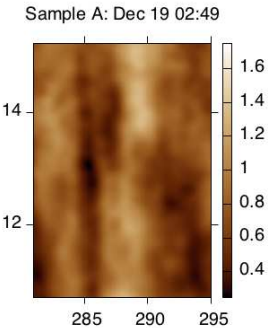

Angle from solar north $(\mathrm{deg})$

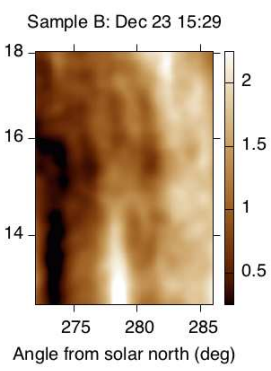

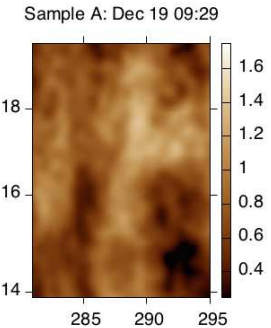

Angle from solar north (deg)

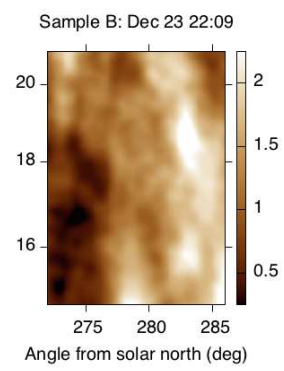

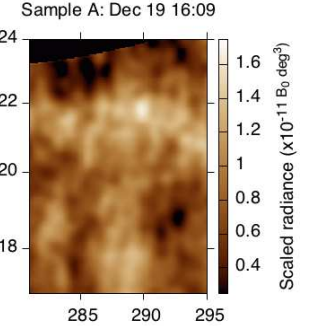

Angle from solar north (deg)

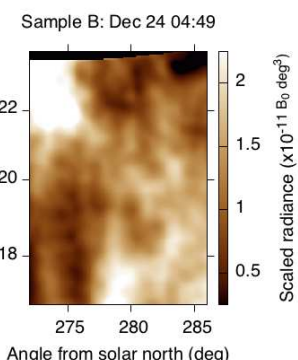

Figure 4. Evolution sequences within two "quiet" striae (radial structures) show fading with distance. The sequences were extracted as in Figure 3, but in locations with no clear puff. See also the corresponding movies in the digital version of this article.

set $\Delta t=0$ and average over $t$, to arrive at a four-dimensional time-averaged structure function $\left\langle S_{x y}\right\rangle$, which characterizes the average local variability of the dataset as a function of location and scale:

$$
\left\langle S_{x y}\right\rangle(x, y, \Delta x, \Delta y) \equiv\left\langle(h(x, y, t)-h(x-\Delta x, y-\Delta y, t))^{2}\right\rangle,
$$

where the angle brackets represent averaging over time. We carried out the computation of the $\Delta t=0$ cut of $S_{x y t}$ at each time and averaged the result across all HI1 frames, prepared as in the middle panel of Figure 2, from 2008 Dec 15 to 2008 Dec 29, to yield $\left\langle S_{x y}\right\rangle$ throughout the dataset with im- age $x$ ranging over azimuthal angle $\alpha$, and image $y$ ranging over $\log (\varepsilon)$. To further reduce noise, we smoothed the computed values of $\left\langle S_{x y}\right\rangle$ by convolution in the $x$ and $y$ dimensions with a kernel $2.7^{\circ}$ wide in $\alpha$ and having the equivalent size in $\log (\varepsilon)$. Further, to analyze radial evolution of "typical" striae and other wind features without contamination from the potentially anomalous central stripe that is visible at $\alpha=270^{\circ}$, we selected two azimuths, $\alpha=250^{\circ}$ and $\alpha=290^{\circ}$ on opposite sides of the $270^{\circ}$ line, and averaged $\left\langle S_{x y}\right\rangle$ between them. This further reduced the dataset to 3 dimensions:

$$
S\left(\varepsilon, \Delta \alpha, \Delta(\log (\varepsilon)) \equiv \overline{\left\langle S_{x y}\right\rangle\left(\left\{250^{\circ}, 290^{\circ}\right\}, \varepsilon, \Delta \alpha, \Delta(\log (\varepsilon))\right),}\right.
$$




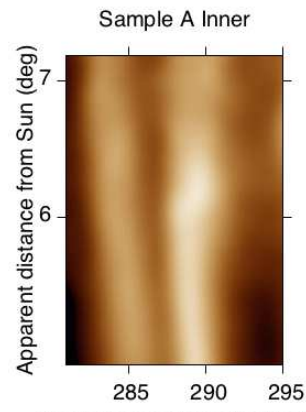

Angle from solar north (deg)

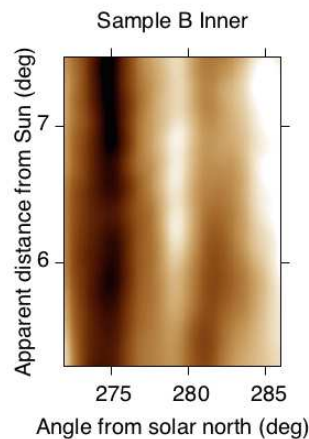

Sample A: vert. smooth

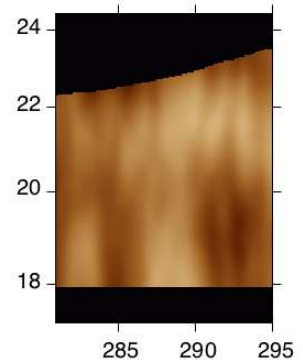

Angle from solar north $(\mathrm{deg})$

Sample B: vert. smooth

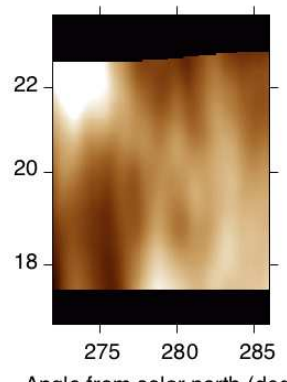

Sample A: horiz. smooth

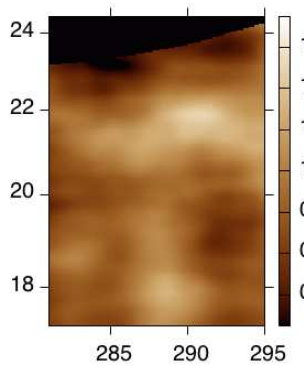

Angle from solar north (deg)

Sample B: horiz. smooth

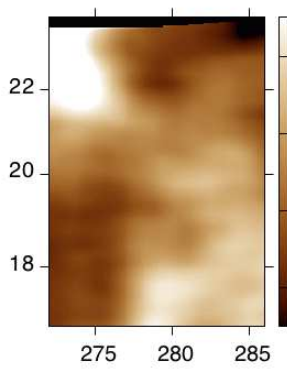

Angle from solar north (deg)
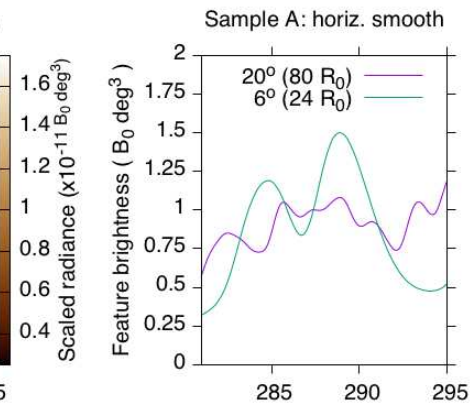

Angle from solar north (deg)

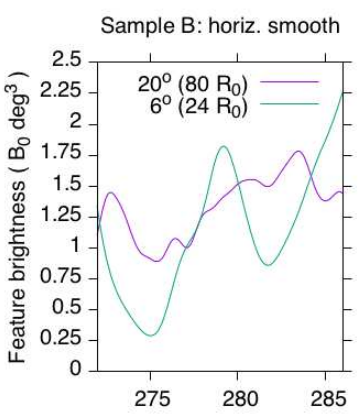

Angle from solar north $(\mathrm{deg})$

Figure 5. Smoothing the final panels of Figure 3 reveals remnant striations even beyond $20^{\circ}\left(80 R_{\odot}\right)$. LEFT: The striations at low altitude, from Figure 4 . MIDDLE: both vertical and horizontal smoothing reveal continued vertical/radial structure below the noise floor. RIGHT: plots of the structure amplitude show $3 \times-5 \times$ attenuation of the striations' measured normalized brightness between $6^{\circ}\left(24 R_{\odot}\right)$ and $20^{\circ}\left(80 R_{\odot}\right)$. Uncertainty may be estimated from the residual high spatial frequencies, as approximately $\pm 0.15 \mathrm{~B}_{\odot} \mathrm{deg}^{-3}$ in each $1^{\circ}$ wide spatial interval.
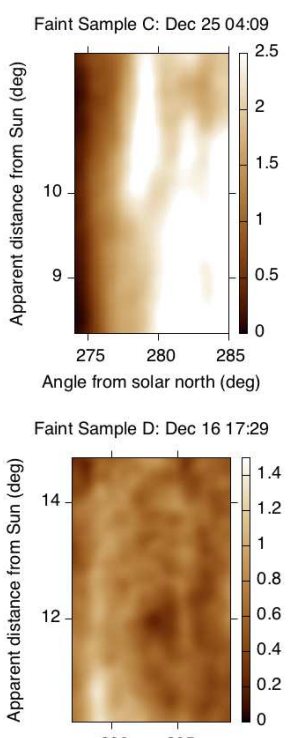

$290 \quad 295$

Angle from solar north (deg)

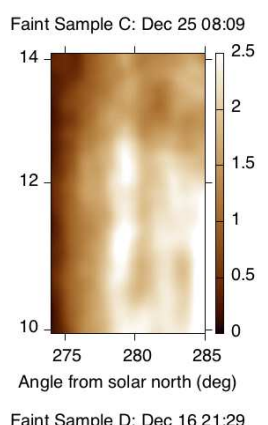

Faint Sample D: Dec 16 21:29

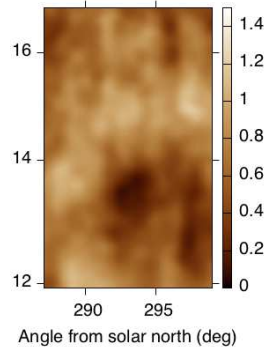

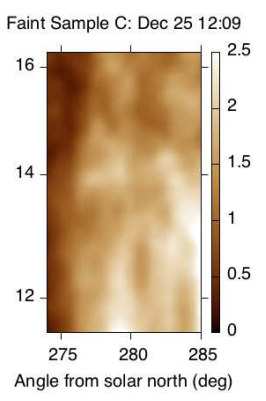

Faint Sample D: Dec 17 01:29

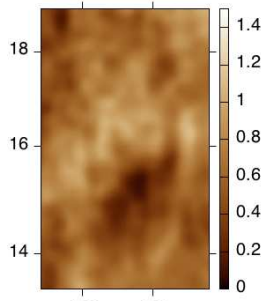

$290 \quad 295$

Angle from solar north (deg)
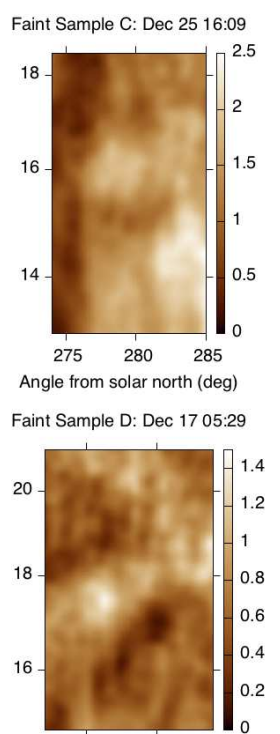

$290 \quad 295$

Angle from solar north (deg)

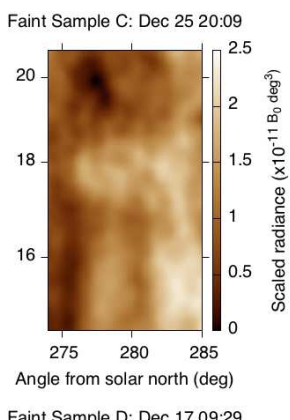

Faint Sample D: Dec 17 09:29

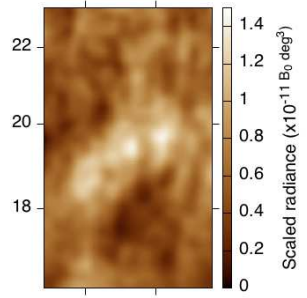

$290 \quad 295$

Angle from solar north (deg)

Figure 6. Faint relatively compact features ("flocculae") grow by comparison to the coronal striae, to generate the flocculated appearance of the outer portion of the HI1 field of view. These faint features (found outside of $15^{\circ}$ from the Sun) appear to "fade in" as they propagate across the field of view. Each sequence shows, from left to right, a sequentially brightening localized image feature subtending approximately $5^{\circ}-9^{\circ}$ of azimuth and roughly vertically centered in the image panel. TOP: a floccule fades in between elongations of $10^{\circ}$ to $18^{\circ}$, even as its host stria fades out. BOTTOM: a floccule fades in between elongations of $13^{\circ}-19^{\circ}$, in isolation from strong striae. 
where the overbar represents the averaging over neighborhoods in $\alpha$ and $\varepsilon$. We used the two separate values of $\alpha$ to symmetrize $S$ as a function of $\Delta \alpha$, while still eliminating the structure near $270^{\circ}$.

$S$ thus contains a separate image in the independent variables $\Delta \alpha$ and $\Delta \varepsilon$ for each separate value of $\varepsilon$. Several of these structure images are shown in Figure 7 . The transformed data use a logarithmic scaling for $\varepsilon$, so these corresponding structure images are most naturally measured in degrees of azimuth and decibels $(\mathrm{dB})$ of elongation angle. One $\mathrm{dB}$ corresponds to a factor of roughly 1.26 in $\varepsilon$, so that in the $\varepsilon=11^{\circ}$ panel at center of Figure 7, a value of $0.8 \mathrm{~dB}$ in the radial separation corresponds to a separation of $2.2^{\circ}$. Because the underlying images are conformal to the original sky plane, the structure function planes are also conformal and any apparent anisotropy reflects a real anisotropy in the original images.

Each image plot across the top row of Figure 7 shows a single 2-D slice of $S$ : it is the squared difference (averaged across azimuthal selections and time) between the value of a single pixel at a given apparent radius and each selected azimuth, and the corresponding value of nearby pixels with the corresponding offset throughout the plotted image. The image values drop to zero at the origin, because the average squared difference between a single pixel and itself is identically zero. The tall, narrow structure of the trough at central radii of $5.7^{\circ}, 8.1^{\circ}$, and $11^{\circ}$ reflects the striation of the "typical" corona at those altitudes: there is more variation of image value in the $\alpha$ direction than in the $\varepsilon$ direction. The more rounded structure at central radii of $15^{\circ}$ and $20^{\circ}$ reflects the increasing isotropization of the brightness variation, at higher altitudes.

The plots in the bottom row of Figure 7 are horizontal and vertical cuts through the origin of the 2-D structure function slices. They reveal the increasing isotropy of the image as the wings of the vertical cuts rise with altitude and the wings of the horizontal cuts drop with altitude. It is immediately apparent by inspection that the shift in the structure function is not due to residual noise in the images: uncorrelated noise would produce a sharp rise from the origin, rapidly transitioning to a shallower slope at scales dominated by image features rather than by background noise. The cuts grow monotonically with distance from the origin, and show no sign of a noise-related break at the scales of interest. The horizontal cuts grow more shallow with altitude, indicating fading of the radial striae, even as the radial cuts grow steeper.

Note that we have followed the typical definition of $S$, which is asymmetric rather than being symmetrized around a central point. This means that $S$ can in principle be, and in practice is, asymmetric with respect to the $\Delta$ variables at particular locations. For example, there is a slight systematic asymmetry visible in the vertical (radial) structure functions in Figure 7. This is because there is a systematic variation in the structure function with $\varepsilon$, and point pairs taken in the positive-going $\Delta \varepsilon$ direction are centered at a different location than point pairs taken in the negative-going $\Delta \varepsilon$ direction.

To better understand the radial evolution, we also plotted the structure function in quasi-spatial coordinates. These are plotted in Figure 8. We made use of the known but nontrivial observing geometry to make the conversion, which is somewhat approximate. In particular, there is a variable $( \pm 5 \%$ to $\pm 40 \%$ ) cross-scale mixing effect, increasing with $b_{\text {eff }}$ across the field of view, due to the shifting perspective along varying lines of sight. Further, because we are observing a large fraction of the corona rather than a compact feature such as a CME, the conversion from elongation $\varepsilon$ to spatial distance from the Sun uses an "effective impact parameter" $b_{e f f}$, rather than the simple trigonometric impact parameter $b$, for each line of sight. Both of these effects are described at length in the Appendix.

While the horizontal (azimuthal) structure function in the images clearly expands faster than the overall radial expansion of the solar wind (Figure 7), the vertical (radial) structure function in the images appears roughly constant as a function of $b_{\text {eff }}$. Figure 9 shows the evolving trend. Because, as we demonstrated in Section 3.1, the average scaled brightness of the wind does not change with altitude, the separation distance $\Delta b_{e f f, t h}$ for $S$ to exceed a set threshold $S_{t h}$ is a valid measure of the changing hardness of $S$ with solar distance. That is plotted in Figure 9, for $S_{t h}=0.5 \times 10^{-22} B_{\odot}^{2} \mathrm{deg}^{6}$. Throughout the measurable range of offsets from the Sun, this thresholding distance remains approximately constant: the radial cuts do not soften as do the lateral cuts. This behavior is consistent with fully developed turbulence in the radial direction.

\section{DISCUSSION}

We have identified and characterized a fundamental change in the apparent texture of the outflowing plasma above the top of the solar corona. Between about $10^{\circ}\left(\sim 40 R_{\odot}\right)$ and about $20^{\circ}\left(\sim 80 R_{\odot}\right)$, the familiar radial striae of the solar corona, which comprise the streamers, pseudostreamers, plumes, and other radial structure in the corona, gradually fade until their brightness is dominated in the slow solar wind $20^{\circ}$ from the Sun by more compact, nearly isotropic features ("flocculation"). We have traced individual features in the flocculated visual field and found that, unlike the Sheeley blobs and Viall puffs previously studied by several other groups, which clearly originate in the corona (e.g., Sheeley et al. 1997; Sheeley \& Rouillard 2010; Viall et al. 2010; Viall \& Vourlidas 2015), these flocculae largely "fade in" over the course of several apparent degrees of propagation above $10^{\circ}$ from the Sun. Both types of visual feature co-exist in the outer field and could represent either different physical phenomena or different parts of a single distribution of fluctuations in the solar wind.

\subsection{Fading of the Striae}

The striae themselves fade faster with respect to radius than would be expected from bright features in a constant-speed, conservative solar wind, eventually dropping below our processed images' noise floor-where they can be seen by further, more aggressive spatial averaging of the HI1 images to further beat down the noise floor, at the cost of further reducing spatial resolution. There is no large-scale breakup of the striae as might be expected from large-scale instabilities.

We are confident in this fundamental observational result, because we have eliminated the most plausible observational scenarios that might mimic fading of the coronal striae. In particular, by demonstrating that the striae, while faint, can be observed even under the reduced noise floor from processed HI1 data, we have shown that noise and background effects alone are not responsible for the apparent fading of the striae - and this result is corroborated by the opposite brightness trend in the flocculae, which have similar spatial scales and scaled brightness values to the striae themselves.

The striae are rendered visible by Thomson scattering, and therefore the features' geometry relative to the Thomson surface-the locus of the sphere whose diameter extends from the observer to the Sun-becomes important. The Thomson 

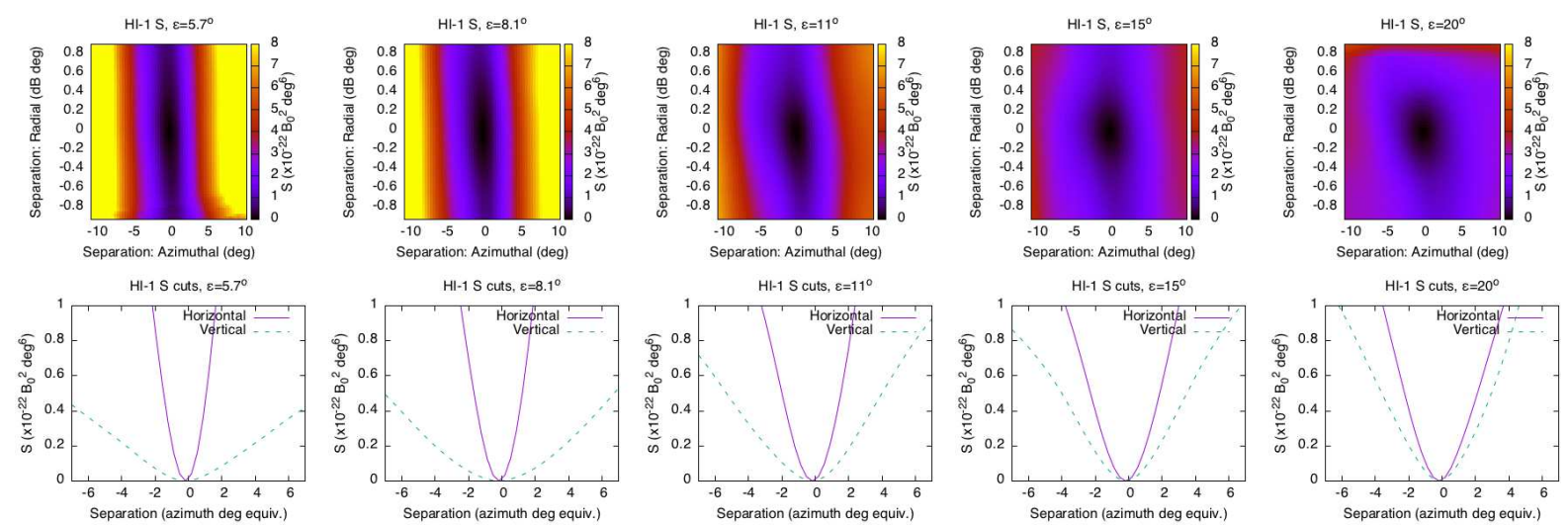

Figure 7. 2-D mage structure functions and their cuts, averaged the entire processed HI1 dataset from 2008 Dec 15 to 2008 Dec 29, reveal the isotropization of image texture with increasing altitude. See text for discussion.
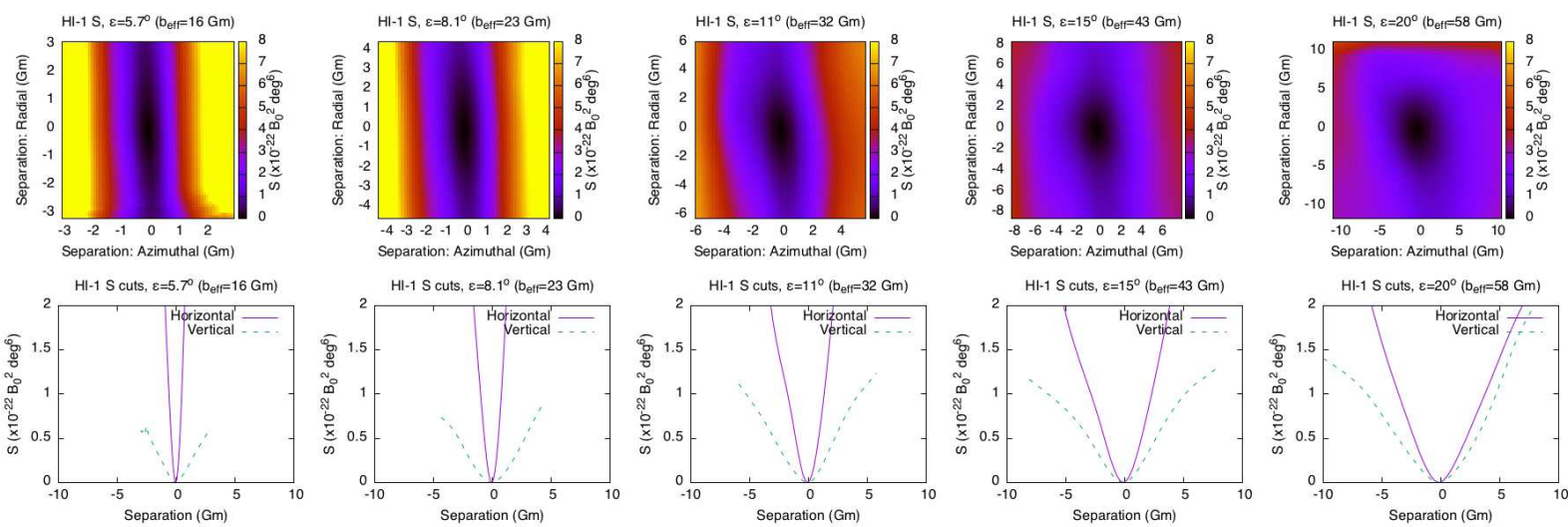

Figure 8. 2-D quasi-spatial structure functions and their cuts, averaged across the entire processed HI1 dataset from 2008 Dec 15 to 2008 Dec 29 , reveal shifts in the solar wind variability with radius from the Sun.

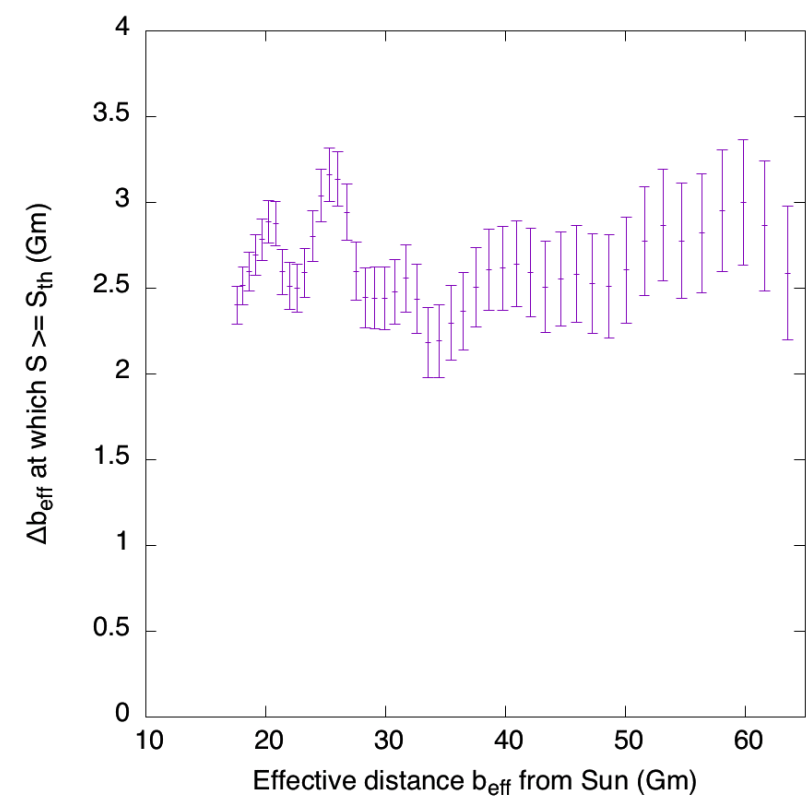

Figure 9. Plot of the separation distance $\Delta b_{\text {eff }}$, for the radial cut of the structure functions in Figure 8 to exceed a threshold $S_{t h}=0.5 \times 10^{-22} B_{\odot}^{2} \mathrm{deg}^{6}$, measures softening of the structure function with altitude. The radial structure function of the images grows neither harder nor softer across the effective altitude range of this study. Error bars are based on a one-image-pixel offset in $\Delta b_{\text {eff }}$. surface marks the point of greatest apparent surface brightness per unit density along a given line of sight, and therefore purely radial structures may gain or lose apparent surface brightness relative to the applied $\varepsilon^{-3}$ scaling, depending on this geometry. In fact Vourlidas \& Howard (2006) proposed to use this effect to locate features in 3-space by looking for anomalous dimming as the features propagate. However, the Thomson surface is surrounded by the "Thomson plateau" (Howard \& DeForest 2012): a broad range of out-ofimage-plane angles over which feature radiance is very nearly independent of geometry, on a given line of sight.

We eliminate the possibility that the fading is due to scattering physics, by noting that no striae appear to fade in with altitude, only to fade out. Because our dataset spans over half a solar rotation and striae are observed to exist across a wide range of coronal longitudes, Thomson plateau effects should cause cross-fading between different striae and not a uniform attenuation of all striae. That is because, in the overall population of dense radial features, approximately the same number of radial features should be outside the Thomson plateau at low altitudes and enter it at high altitudes, as exist within the Thomson plateau at low altitudes and exit it at high altitudes. Moreover, because the field of view is smaller than the angular extent of the Thomson plateau, it is expected that most visible striae remain within the plateau for their entire visible length.

Turning to more subtle solar geometric effects: the "pancake effect" of azimuthal expansion in the solar wind, coupled with perspective/projection effects, can cause fading of 
features that are compact in the lower corona and separated radially. Features of that description can apparently merge in the image plane as they propagate outward, even when no physical merging or mixing occurs; this is illustrated with a simple geometric argument by Viall et al. (2010). Similar effects can cause apparent merging in blobs that pass one another along the line of sight, as quantified for the 'Sheeley blobs' by Sheeley \& Rouillard (2010). These effects impact variations along the radial direction and may affect the "flocculated" appearance. Importantly for the current work, these effects work to blend individual features and produce an illusion of a radial stria, rather than the opposite, so they cannot account for the general fading of the striae. To cause a stria to fade, this mechanism requires a very rare coincident coalignment to cause an illusive stria close to the Sun and reveal puffs farther from the Sun.

The fading of the striae, in the scaled brightness parameter $B_{\odot} \mathrm{deg}^{3}$, is surprising because it varies from expected behavior of a mass-conserving, constant-speed radial solar wind. The two most obvious physical mechanisms to explain it are (A) that the wind might not be constant speed, or (B) that the features themselves might not conserve visible mass.

We eliminate acceleration effects (possibility A) by noting that, to explain the observed fade of a factor of more than 3 in scaled brightness, the wind inside the striae would have to accelerate by a comparable factor across the field of view. No such acceleration is measured, and the outflow speed of bright features varies by less than a factor of 2 across the entire image (and considerably less along a given radius).

Turning to conservation effects (possibility B): conservation of bright structure is not necessarily conservation of mass. The images are produced using sunlight that is Thomson-scattered off of free electrons. Photometric mass estimates in the heliosphere (DeForest et al. 2014) or corona (Vourlidas et al. 2000) are produced by assuming the free electrons are part of a neutral, 100\% ionized plasma with approximately coronal composition (specifically, proton-to-helium ratio). Recombination of ions and free electrons could in principle cause the striae to fade, but this effect would require more than $2 / 3$ of the plasma to neutralize. That effect is implausible: although electrons in the solar wind typically suffer a few tens of collisions enroute from the Sun (Salem et al. $2003)$, by the altitude range under consideration $\left(40-100 R_{\odot}\right)$, collisions are very rare (Hundhausen et al. 1968; Owocki \& Scudder 1983). Further, the high kinetic temperature of order $10^{5}-10^{6} \mathrm{~K}$ prevents recombination (Gibson 1973).

Eliminating (A) and recombination effects, leaves the possibility of intrinsic variation of the entrained mass within each stria. Such mass loss would be due to diffusion or other effects that transport material from the dense structure to the intervening spaces.

One possibility for structural mass loss is misalignment, at high altitudes, of quasi-planar structures that may, by coincidence, align with the line of sight when seen closer to the Sun. This possibility is important because many streamers and pseudostreamers are thought to have quasi-planar geometry rather than being compact structures (Wang et al. 2007). But the same argument applies to these effects as to effects from the geometry of Thomson scattering: if the striae were primarily due to chance alignments with quasi-planar density structures (and loss of alignment were the reason most observed striae fade at large radii), then chance alignments at those farther distances should be expected to cause a roughly equal number of striae to fade in as out - which is not observed. We conclude that the fading striae are not due to chance alignments and subsequent misalignments of smoothly expanding quasiplanar density structures in the solar wind.

Our discussion so far has focused on eliminating various observational effects that could in principle explain the apparent radial evolution seen in the images and diagnostic parameters shown in Section 3. It remains to offer a physical scenario to explain the fading of striations and emergence of puffs and flocculation, and the isotropization of the computed structure functions of the images. We now consider the effects of the turbulent fluctuations observed at $1 \mathrm{AU}$, extrapolated inward to the observed range of altitudes, and whether they can account for the inferred lateral transport of mass.

We begin by recalling the general changes in nonlinear behavior moving outward from the Sun. Below the Alfvén surface where the solar wind speed exceeds the radial speed of fast-mode MHD waves, the plasma is also low $\beta$ and dynamics are strongly influenced by the magnetic field. This gives rise to a strong correlation (or spectral) anisotropy relative to the magnetic field direction (Zank \& Matthaeus 1992; Cranmer et al. 2015; Oughton et al. 2015). Under these conditions, magnetic flux tubes strongly align with the large scale magnetic field, and magnetic fluctuation gradients concentrate in the perpendicular direction. The variability of density across flux tubes then gives rise to strong anisotropic density gradients that, in turn, appear as striae in the imaging data. In the sub-Alfvénic coronal regions, the dynamical emergence of this anisotropy is in part due to active anisotropic spectral transfer (Shebalin et al. 1983; Oughton et al. 1994) that amplifies the transverse gradients relative to the radial ones. Furthermore, in the corona this turbulence is possible because inward propagating fluctuations overcome the outward flow of the solar wind and interact with their outward propagating counterparts (MHD wave-wave interactions).

Above the Alfvén surface, both of these effects change. First, inbound (in the co-moving frame) waves are advected outward (in the solar system stationary frame), reducing the overall strength of the wave field and delaying development of active Alfvénic turbulence. Second, above the related $\beta=1$ surface the magnetic field strength is no longer dominant over either thermal or convective effects. On the other hand, the state of the plasma flowing into this critical region near the Alfvén and $\beta=1$ surfaces is already highly anisotropic, having been shaped by the well-ordered and nearly radial average magnetic field. This accounts for the dominant appearance of striae, interrupted by discrete features including CMEs, etc., in images from coronagraphs and our HI1 dataset. Therefore, the observed striae seen at or above the $\beta=1$ surface are consequences of factors operating at lower altitudes. Although the striae persist into our field of view, the factors that build and maintain the coronal anisotropy are no longer present in the young solar wind.

One may now ask whether the existing turbulence field in the solar wind at $150 \mathrm{Gm}(1 \mathrm{AU})$, extrapolated inward to $38 \mathrm{Gm}$ ( $b_{\text {eff }}$ for lines of sight close to $12^{\circ}$ from the Sun), account for the breakup of the striae that we observe there. At $150 \mathrm{Gm}$, typical values of the turbulent velocity $Z(150 \mathrm{Gm})$ and correlation length $L(150 \mathrm{Gm})$ are $25 \mathrm{~km} \mathrm{~s}^{-1}$ and $1 \mathrm{Gm}$, respectively (Ruiz et al. 2014; Isaacs et al. 2015). Both of these quantities are approximately log-normally distributed, so that extreme outliers are to be expected. Observations suggest that $Z \propto r^{-1 / 2}$ in the inner heliosphere (Verma \& Roberts 
1993), so that $Z(38 \mathrm{Gm}) \approx 12 \mathrm{~km} \mathrm{~s}^{-1}$. Further, taking the correlation length to scale between $L \propto r$ and $L \propto r^{1 / 2}$ (Breech et al. 2008), at $38 \mathrm{Gm}(0.25 \mathrm{AU})$ the correlation scale should be $L(0.25 A U) \approx 0.25 \mathrm{Gm}-0.5 \mathrm{Gm}$ if the turbulent field observed at $150 \mathrm{Gm}(1 \mathrm{AU})$ persists inward to this extent.

Based on the estimates above, correlated density or flow features in the outer reaches of the solar wind observed in Figure 2 have an estimated expected size of $0.25-0.5 \mathrm{Gm}$, and subtend approximately $0.25^{\circ}-0.5^{\circ}$ of azimuth. Features of this scale are close to the resolution limit of the present observation, but large-scale outliers, extending to several times the expected correlation scale, should be observed if the the nearEarth turbulent field happens to be already well developed in the observed range of altitudes.

In fact, such displacements appear to be observed. Figure 10 shows the lateral evolution of the stria in the bottom row of Figures 4 and 5 as it propagates outward; this stria is typical and several other striae show similar behavior. The image shows the average brightness evolution, averaged vertically across $10 \%$ of image height, in a horizontal band extending across each of the subfield images in the Figure 4 dataset. The overlain points reveal lateral evolution of the center of the stria in the co-moving frame of the solar wind. Each point shows the horizontal location of maximum brightness at the corresponding time and apparent distance (elongation $\varepsilon$ ). We determined the error bars by noting that the RMS noise level at $\varepsilon=14^{\circ}$ is $4 \times 10^{-13} R_{\odot} \mathrm{deg}^{3}$, and finding the location where the radiance in each horizontal cut falls from the maximum by at least that amount. The points are calculated only for every third frame of the dataset, to avoid the effects of the three-frame temporal averaging we used during the processing: each point represents a true independent sample.

While the variations in azimuth do not exceed the error bars for most pairs of points, the coherence of the lateral motion renders the motion statistically significant. The amplitude is approximately $0.5^{\circ}$ in azimuth, in good agreement with the estimate above.

The lateral motions that we observe are inconsistent with direct radial motion and consistent with either scaling of the fully developed turbulence detectible near Earth, with local onset of lateral turbulent motion due to hydrodynamic instabilities (such as the Kelvin-Helmholtz instability), or with isotropization of MHD turbulence present in the corona itself. Lateral convective transport from these processes must occur to account for the fading of the striae, but there is no clear and direct sign of the postulated breakup. Therefore, we conclude that the breakup must occur on scales smaller than the effective spatial resolution of $\sim 0.5-2.5 \mathrm{Gm}$.

\subsection{Onset of the Flocculation}

In counterpoint to the fading of the striae, flocculae "fade in" smoothly from elongation angles of $\sim 8^{\circ}$ out to $20^{\circ}$. The radial length scale of the flocculae is comparable to the length scale in the Viall puffs and/or Sheeley blobs that enter the HI1 field from the lower corona: 3-10 Gm. Two explanations spring immediately to mind: (1) the flocculae could be local interaction regions: zones of density enhancement caused by variation of outflow speed along a given wind flow streakline $^{6} ;(2)$ the flocculae could be a symptom of the same insta-

\footnotetext{
${ }^{6}$ Readers are reminded that a streakline is the path in space traced by a particular test particle in a time-dependent flow field; this is distinguished from a streamline, which is the path in space traced by an incompressible flow field at a particular moment in time.
}

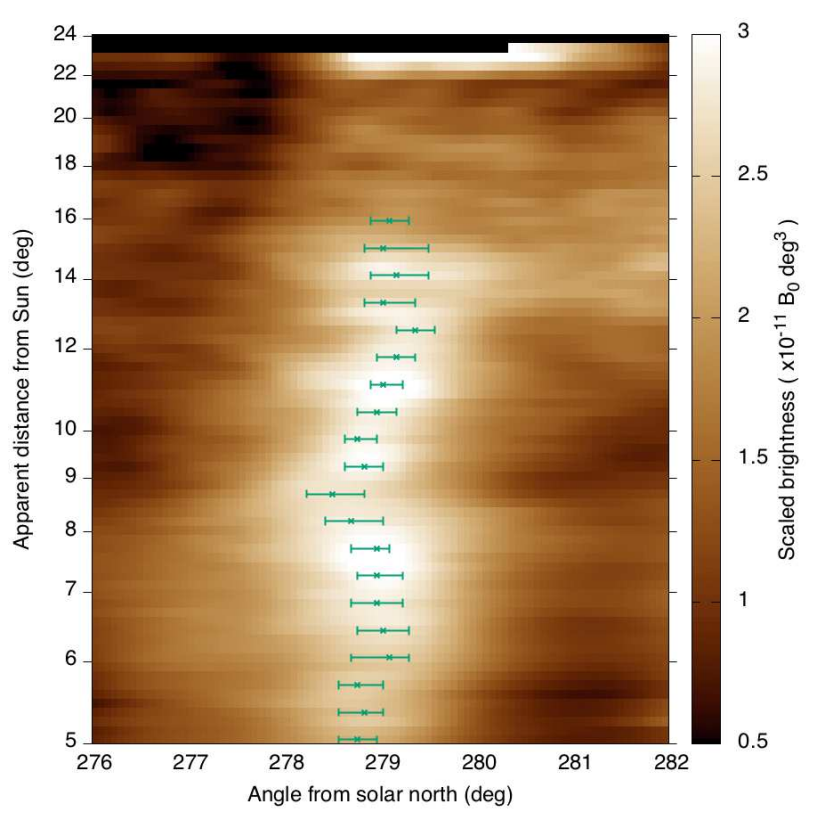

Figure 10. Co-moving evolution plot shows brightness in a horizontal cut through the stria in the bottom row of Figures 4 and 5, in a co-moving frame rather than at a single time. The overlain curve and error bars indicate the location of the brightest portion of the stria. The apparent shifts in azimuth are marginally inconsistent with pure radial motion, suggesting lateral perturbations to the outward motion. See text for details.

bilities that are seen to offset and fade the striae.

To investigate the hypothesis that the flocculae are caused by local interactions between different-speed streams, we consider the divergence of the velocity field $\nabla \cdot \mathbf{v}$ necessary to cause the observed growth rate of the density in particular flocculae (as in Figure 6). To determine this divergence, we must estimate the proportional density enhancement represented by the brightness growth of the flocculae.

Because of the way that coronal and heliospheric images are created, there is no "absolute zero" to unpolarized Thomson scattering images in general. This is because the images are produced by subtracting a steady or nearly-steady background model in the fixed focal plane (coronagraphs) and/or the Celestial sphere (heliospheric images); and there is no intrinsic difference between a steady signal from the $F$ corona or stray light, and a steady signal from a stationary or quasi-stationary electron density structure in the field of view. Hence, essentially all unpolarized Thomson scattered images from current instruments report "feature excess" brightness, and hence inferred densities are only "feature excess" densities, compared to an unknown steady background.

Near Earth, the typical electron density of the slow solar wind is $3-10 \mathrm{~cm}^{-3}$. Extrapolating inward to $10^{\circ}$ elongation from the Sun (a line of sight angle whose weighted average distance from the Sun is $28 \mathrm{Gm}$; see the Appendix), we estimate that the "typical background" electron density at $10^{\circ}$ elongation in our images is $100-300 \mathrm{~cm}^{-3}$, along an effective line-of-sight distance of $50 \mathrm{Gm}(0.33 \mathrm{AU})$. Taking the lower figure and following Equation 5 of Howard \& DeForest (2012), we infer that

$$
B_{b k}\left(\varepsilon=10^{\circ}\right) \approx \frac{B_{\odot} \sigma_{t} \pi r_{\odot}^{2}}{\left((1 A U)\left(\sin \left(10^{\circ}\right)\right)^{2}\right.}\left(100 \mathrm{~cm}^{-3}\right)(50 \mathrm{Gm}),
$$

where $B_{b k}$ is the calculated background radiance from Thomson scattering, $B_{\odot}$ is the mean solar radiance, $\sigma_{t}$ is the Thomson scattering cross section of $4.0 \times 10^{-30} \mathrm{~m}^{2}$, and $r_{\odot}$ 
is the mean solar radius of $6.96 \times 10^{8} \mathrm{~m}$. This evaluates to $B_{b k}\left(\varepsilon=10^{\circ}\right)=4.2 \times 10^{-14} B_{\odot}$. Multiplying by $\varepsilon^{3}$ yields the elongation-independent scaled background radiance $B_{b k}^{\prime}=$ $4.2 \times 10^{-11} B_{\odot} \mathrm{deg}^{3}$. This $B_{b k}^{\prime}$ remains approximately equal throughout all values of $\varepsilon$ that we observed.

$B_{b k}^{\prime}$ is comparable to the measured feature-excess brightness in typical flocculae as seen in the rightmost panels of Figure 6. The calculated $B_{b k}^{\prime}$ exceeds this feature brightness by a factor of 3-10. However, a typical floccule does not extend along the entire line of sight. Taking the flocculae in Figure 6 to have roughly the same extent out of the screen as in azimuth, they subtend roughly $10^{\circ}$ relative to Sun center, rather than the approximately $78^{\circ}$ subtended by the effective line of sight-and therefore the excess density associated with each floccule is larger than the calculated ratio of $B_{\text {floccule }}^{\prime}(\varepsilon) / B_{b k}^{\prime}$ by a factor of $\sim 8$. Hence the floccule's excess density is roughly equal to the density of the background flow at the same positions on the line of sight, at the highest elongations in Figure $6\left(\sim 20^{\circ}\right.$ from the Sun). This implies that, between altitudes of roughly $40 R_{\odot}$ and $80 R_{\odot}$, the density in a floccule approximately doubles compared to quiescent propagating wind.

The flocculae have typical radial sizes of 3-6 Gm, so if the density enhancement arises from a simple negative divergence of the bulk flow, the material at the visible edges must propagate inward relative to the mean bulk flow, crossing the width of the floccule during a transit across $40 R_{\odot}(30 \mathrm{Gm})$. Thus, the average $\Delta v$ between the leading and trailing sides of the floccule must be about $10 \%-20 \%$ of the wind speed, corresponding to a $\pm 5 \%$ to $\pm 10 \%$ antisymmetric deviation from the mean radial outflow speed in the vicinity of each floccule.

In light of the existence of highly structured radial striae, cross-field evolution due to instabilities or onset of turbulence must play a large role in the development of the flocculae. If the flocculae were generated by purely compressive processes due to temporal variation in the speed of individual flux tubes' coronal wind, as described above, then their azimuthal size should be comparable to the observed scale of individual wind structures-i.e., striae. Instead, we observe the flocculae to be, typically, over 3 times larger in azimuthal extent than are typical striae. This implies collective behavior on scales larger than the natural azimuthal scale on which the wind is injected from the corona itself.

An alternative explanation is that the flocculae are density enhancements caused by turbulent perturbations to the flow field rather than by pileup in a smooth flow. In this view, turbulent motions within the flow give rise to density perturbations on length scales comparable to the correlation scale of the turbulence. We note, however, that the radial length scale of the flocculae is about $10 \times$ larger, and their azimuthal length scale is perhaps $30 \times$ larger, than the scaled correlation length calculated in Section 4.1. In this regard, one might explain the flocculae as density enhancements associated with the very largest and strongest velocity perturbations, associated with the onset of turbulence in the inner heliosphere. The moderately frequent occurrence of dynamically active fluctuations appears to be reasonable, based on the observed long tails on the distributions of correlation lengths (approximately log normal) seen in Helios data (Ruiz et al. 2014). It is also possible that the flocculae emerge as a manifestation of so called " $f^{-1}$ " noise, which is observed in situ at very low frequencies in solar wind density observations (Matthaeus et al. 2007).
A detailed analysis of how and whether exceptional turbulent fluctuations, $f^{-1}$ noise, or some other collective phenomenon may account for the present observations is a topic for future work.

\section{CONCLUSIONS}

We have imaged, above the solar corona, a fundamental shift in texture of the solar wind: from the highly anisotropic, magnetically structured coronal plasma to more isotropic, flocculated solar wind plasma. We have eliminated several of the more common sources of systematic error, including image degradation due to the weaker signal at increasing solar elongation, and conclude that the transition is real. We observed the ultimate disposition of the radial striae that, aside from the rapid falloff as the cube of elongation angle $\varepsilon^{3}$, are the most prominent visual feature of the outer corona. Further, we have noted and described the simultaneous development of flocculae: local density enhancements that arise in the outflowing solar wind as the striae disappear. These two effects together combine to radically change the visual texture between the outer corona and the inner heliosphere, and mark the profound shift between the primarily magnetically structured corona and the primarily hydrodynamic solar wind.

Moreover, the shift in texture points strongly to the early development of a turbulent cascade, of which we can observe primarily the energy-bearing and largest inertial scales. The behavior of the features is consistent with onset and development of quasi-isotropic turbulence due to hydrodynamic and MHD instabilities, and inconsistent with smooth flow.

The striae fade gradually at resolutions attainable with the HI1 measurement at apparent distances (elongation angles) of $10^{\circ}-20^{\circ}$ from the Sun, corresponding to $44-88 R_{\odot}$ in effective line-of-sight impact parameter $b_{\text {eff }}$. In that interval they typically fall in brightness by a factor of $\sim 3-5$ compared to the behavior expected for a smooth, unperturbed solar wind. In that same interval, they undergo slight non-radial distortions that, while only marginally detected, are prima facie consistent with the large-scale tail of a forming turbulent cascade whose parameters scale correctly to the observed variability of the slow solar wind $150 \mathrm{Gm}$ (1 AU) from the Sun. The striae, while faint, still exist at the outer edge of the HI1 field of view (solar elongation of $24^{\circ}$, off the centerline of the images), and become visible with broader smoothing of the images.

In approximately the same altitude range that the striae fade, localized high-density puffs of solar wind (which we term "flocculae") develop gradually, fading into existence without noticeable lateral spreading or internal motion as they brighten. The flocculae are consistent with variations in local wind speed of the order of $\pm 5 \%-15 \%$, but require collective motion to form at the azimuthal size scales observed. This, too, may be a signature of either developing turbulence or (equivalently) the early onset and action of large-scale hydrodynamic instability (e.g., Ofman 2016).

The disappearance of the striae, and appearance of the flocculae, are not due to Thomson surface projection effects. The entire population of striae fades out, and the entire population of flocculae fades in; Thomson effects would cause some of each type of feature to fade out and others to fade in with altitude, without (on average) changing the total populations. Contrariwise, the nuances of Thomson scattering at high solar altitudes yield a calculated "Thomson plateau" of nearly constant apparent radiance per unit density (Howard \& DeForest 2012). This plateau is quite broad-over $100^{\circ}$ in out-of-plane 
angle relative to the Sun-compared to the field of view of our measurements. Therefore, most striae and most flocculae remain well within the plateau throughout the observed range of apparent distances.

In producing the images, we have demonstrated several novel-to-coronagraphy image processing techniques: minsmooth unsharp masking, time-shifted image co-addition, and structure function analysis to characterize ensemble texture. These techniques are almost certainly useful for related studies well beyond this simple analysis.

This study is close to the limit of what is possible with the existing HI1 instruments. Higher sensitivity, higher resolution (in space and time) images of this transition zone from coronal to heliospheric physics are necessary to reveal whether, in fact, formation and development of a nearly isotropic turbulent cascade is responsible for the loss of coronal structure and development of variable flocculated structure in the solar wind at these large observable scales, to and identify the relative importance of coronal features and instability growth to the structure of the solar wind itself. The upcoming Solar Probe mission will reveal wind structure on fine scales and can measure parameters such as the Alfvén speed, wind speed, and $\beta$ parameter directly at particular locations-but capturing the effect of these transitions and their import for the energy-bearing range of solar wind turbulence will require both these insights and a more global perspective on the structure of the corona to solar wind transition.

C.E.D. is supported by grant NNX15AB72G from NASA's Living With a Star Targeted Research \& Technology program. W.H.M. is supported by the Solar Probe Plus IS $\odot$ IS project, NSF SHINE grant AGS-1156094, the NASA LWS grant NNX15AB88G, and the Heliospheric GCR grant NNX14AI63G. N.M.V. is supported by the NASA's Heliophysics Guest Investigator program. S.R.C. is supported by NSF SHINE grant AGS-1540094, NASA HSR grants NNX15AW33G and NNX16AG87G, and start-up funds from the Department of Astrophysical and Planetary Sciences at the University of Colorado, Boulder. The work was improved by informative discussions with, and acerbic commentary from, T. A. Howard. The authors gratefully acknowledge the STEREO team for making their data available to the public, and the Solar Probe Science Working Group for making it clear that this article was necessary. The analysis relied heavily on the freeware Perl Data Language (http://pdl.perl.org).

\section{REFERENCES}

Aletti, V., Velli, K., Boccialini, K., Einaudi, G., Georgoulis, M., \& Vial, J.-C. 2000: Astrophys. J. 544, 550..

Antiochos, S.K., Mikić, Z., Titov, V.S., Lionello, R., and Linker, J.A. 2011: Astrophys. J. 731, 2.

Bohlin, J.D. 1970: Sol. Phys. 12, 240.

Breech, B., Matthaeus, W.H., Minnie, J., Bieber, J.W., Oughton, S., Smith,

C.W., \& Isenberg, P.A. 2008: J Geophys. Res. 113, 8105.

Brueckner, G.E., et al. 1995: Sol. Phys. 162, 357.

Cohen, O. 2015: Sol. Phys. 290, 2245.

Cover, T.M., and Thomas, J.A. 1991: Elements of Information Theory (Wiley, New York), ISBN 978-047062592, p. 175.

Cranmer, S.R., van Ballegooijen, A.A., \& Edgar, R.J. 2007: Astrophys. J. $171,520$.

Cranmer, S.R., van Ballegooijen, A.A., \& Woolsey, L.N. 2013: Astrophys. J. 767,125

Cranmer, S.R., Asgari-Targhe, M., Miralles, M.P., Raymond, J.C., Stachan, L., Tian, H., and Woolsey, L. 2015: Phil. Trans. Roy. Soc. A, 373, 2041.
DeForest, C.E., Hoeksema, J.T., Gurman, J.B., Thompson, B.J., Plunkett, S.P., Howard, R.A., Harrison, R.C., and Hassler, D.M. 1997: Sol. Phys. $175,393$.

DeForest, C.E. \& Gurman, J.B. 1998: Astrophys. J. 501, L217.

DeForest, C.E., Plunkett, S.P., \& Andrews, M.D. 2001: Astrophys. J. 546, 569.

DeForest, C.E. 2004: Sol. Phys. 219, 3.

DeForest, C.E., Howard, T.A., and Tappin, S.J. 2011: Astrophys. J. 738, 103.

DeForest, C.E., Howard, T.A., and McComas, D.J. 2014: Astrophys. J. 787,

124.

DeForest, C.E., Matthaeus, W.H., Howard, T.A., \& Rice, D.R. 2015: Astophys. J. 812, 108.

DeForest, C.E. \& Howard, T.A. 2015: Astrophys. J. 804, 126.

Domingo, V., Fleck, B., \& Poland, A.I. 1995: Sol. Phys. 162, 1.

Elliott, H.A., Henney, C.J., McComas, D.J., Smith, C.W., \& Vasquez, B.J. 2012: J. Geophys. Res. 117, A09102.

Eyles, C.J. et al. 2009: Sol. Phys. 2009, 254, 387.

Feng, X., Ma, X., \& Xiang, C. 2015: J Geophys. Res (Sp. Ph.), 120, 10159. Gibson, E.G. 1973: The Quiet Sun, NASA publication SP-303 (U.S. Govt. Printing Office, Washington), Stock No. 3300-0454, p. 274.

Gosling, J.T., Jildner, E., MacQueen, R.M., Munro, R.H., Poland, A.I., and Ross, C.L. 1974: J Geophys. Res. 79, 4581.

Gosling, J.T., \& Pizzo, V.J. 1999: Sp. Sci. Rev. 89, 21.

Harrison, R.A., Davis, C.J., \& Eyles, C.J. 2005: Adv. Sp. Res. 36, 1512.

Howard, T.A., Webb, D.F., Tappin, S.J., Mizuno, D.R., \& Johnston, J.C.

2006: J Geophys. Res. (Sp. Phys.) 111, A04105.

Howard, R.A. et al. 2008: Sp. Sci. Rev. 136, 67.

Howard, T.A. \& Tappin, S.J. 2009: Sp. Sci. Rev. 147, 31.

Howard, T.A. 2011: J. Atm. Sol-Terr. Phys. 73, 1242.

Howard, T.A., and DeForest, C.E. 2012: Astrophys. J. 752, 130.

Hundhausen, A.J., Gilbert, H.E., \& Bame, S.J. 1968: Astrophys. J. 152, L3.

Hundhausen, A.J. 1972: Coronal Expansion and Solar Wind, Series: Physics and Chemistry in Space, ISBN 978-3-642-65416-9 (Springer, Berlin).

Isaacs, J.J., Tessein, J.A., and Matthaeus, W.H. 2015: J Geophys. Res., 120, 868.

Karimabadi, H., Roytershteyn, V., Wan, M., Matthaeus, W.H., Daughton, W., Wu, P., Shay, M., Loring, B., Borovsky, J., Leonardis, E., Chapman, S.C., \& Nakamura, T.K.M. 2013: Phys. Plasmas 20, 012303.

Kepko, L., Spence, H.E., \& Singer, H.J. 2002: Geophys. Res. Lett., 29, 080000 .

Kepko, L., Viall, N.M., Antiochos, A., Lepri, S., Kaper, J., \& Weberg, M. 2016: Geophys. Res. Lett. 43, doi: 10.1002/2016GL068607.

Lionello, R., Velli, M., Downs, C., Linker, J., Mikić, Z., \& Verdini, A. 2014: Astrophys. J. 784, 120.

MacQueen, R.M., Eddy, J.A., Gosling, J.T., Hildner, E., Munro, R.H., Newkirk, G.A., Poland, A.I., and Ross, C.L. 1974: Astrophys. J. 187, L85.

Matthaeus, W., Dasso, S., Weygand, J., Milano, L.J., Smith, C.W., \& Kivelson, M.G. 2005: Phys. Rev. Lett. 95, 231101.

Matthaeus, W.H., Breech, B., Dmitruk, P., Bemporad, A., Poletto, G., Velli, M., \& Romoli, M. 2007: Astrophys. J. 657, L121.

Matthaeus, W.H. \& Velli, M. 2011: Sp. Sci. Rev. 160, 145.

McComas, D.J., Phillips, J.L. Hundhausen, A.J., \& Burkepile, J.T. 1991:

Geophys. Res. Lett. 18, 73.

Montomery, D. \& Turner, L. 1981: Phys. Fluids 24, 825.

Newkirk, G., Jr., \& Harvey, J. 1968: Sol. Phys. 3, 321.

Ofman, L. 2016: "MHD Waves in the Solar Wind," in Low-Frequency

Waves in Space Plasmas (eds. A. Keiling, D.-H. Lee, \& V. Nakariakov),

ISBN 978-1119055006 (John Wiley \& Sons Inc., Hoboken, NJ).

Oughton, S., Priest, E.R., \& Matthaeus 1994: J. Fluid Mech. 280, 95.

Oughton, S., Matthaeus, W.H., Wan, M., \& Osman, K.T. 2015: Phil. Trans. R. Soc. A 373, 20140152.

Owocki, S.P. \& Scudder, J.D. 1983: Sol. Phys. 270, 758.

Papazian, C. 2003: The Complete Joy of Home Brewing, 3rd Edition, ISBN 978-0060531058 (HarperResource, New York).

Rappazzo, A.F., Matthaeus, W.F., Ruffolo, D., Servidio, S., \& Velli, M. 2012: Astrophys. J. L. 748, L14.

Raymond, J.C., McCauley, P.I., Cranmer, S.R., \& Downs, C. 2011: Astrophys. J. 88, 152.

Rudin, W. 1987: Real and Complex Analysis (3rd ed.), ISBN 978-0-07-054234-1 (McGraw-Hill Book Co., New York).

Ruiz, M.E., Dasso, S., Matthaeus, W.H., and Weygand, J.M. 2014: Sol. Phys. 289, 3917.

Saito, K. 1965: Publ. Astron. Soc. Japan, 17, 1.

Salem, C., Hubert, D., Lacombe, C., Bale, S.D., Mangeney, A., Larson, D.E., \& Lin, R.P. 2003: Astrophys. J. 585, 1147.

Schulz-Dubois, E.O., \& Rehberg, I., 1981: Appl. Phys. 24, 323. 
Shebalin, J.V., Matthaeus, W.H., \& Montgomery, D. 1983: J. Plasma Phys. $29,525$.

Sheeley, N.R. Jr. et al. 1997: Astrophys. J. 484, 472.

Sheeley, N.R. Jr. \& Rouillard, A.P. 2010: Astrophys. J. 715, 300.

Tomczyk, S. \& McIntosh, S.W. 2009: Astrophys. J. 697, 1384.

Verma, M.K. and Roberts, D.A. 1993: J. Geophys. Res. 98, 5625.

Viall, N.M.,Spence, H.E., \& Kasper, J. 2009:Geophys. Res. Lett. 36, L23102.

Viall, N.M., Kepko, L., \& Spence, H.E. 2009, J Geophys. Res. 114, A01201.

Viall, N.M., Spence, H.E., Vourlidas, A., \& Howard, R.A. 2010: Sol. Phys. $267,175$.

Viall, N.M., \& Vourlidas, A. 2015: Astrophys. J. 2015, 807, 176.
Vourlidas, A., Subramanian, P., Dere, K.P., \& Howard, R.A. 2000: Astrophys. J. 534, 456.

Vourlidas, A. \& Howard, R.A. 2006: Astrophys. J. 642, 1216.

Walker, A.B.C., Lindblom, J.F., Barbee, T.W., \& Hoover, R.B. 1988:

Science, 241, 1781 .

Wang, Y.-M., Sheeley, N.R., \& Rich, N.B. 2007: Astrophys. J. 658, 1340.

Webb, D.F. et al. 2006: J. Geophys. Res. (Sp. Phys.) 111, A12101.

Zank, G.P. \& Matthaeus, W.H. 1992: J. Geophys. Res. 97, 17189.

Zirker, J.B. 1977: Rev. Geophys. Sp. Phys. 15, 257

\section{APPENDIX}

\section{A. GEOMETRY AND SPATIAL SCALES}

Analyzing the spatial structure function of the corona, as sampled by our images, requires converting the scaled brightness images from their native angular coordinates to distance units in the corona. This is non-trivial, because the scales sampled by the image and its structure function in the vicinity of a given pixel are sampled in observer-centered angular coordinates, relating variation of the brightness along an integrated line of sight to that line of sight's azimuth and elongation angles $(\alpha, \varepsilon)$ relative to the observer and Sun. In contrast, the structure function of interest to understand turbulent onset is sampled in spatial coordinates, relating variation in density (or its proxy, Thomson-scattering emissivity) to spatial offset, as a function of radial distance $r$ from the Sun. The various angles and distances are shown in Figure 11; and $\alpha$ is considered to be an angle of revolution about the observer-Sun line.

For this initial treatment of structure function variation, we treated the neighborhood of a structure function sample as a collection of plane-parallel lines through a finite locus of corona, to approximate the more complex geometry of the sampling. This introduces some "slop" in the scales represented by sample offsets $(\Delta x, \Delta y)$ in the spatial structure function, and in the radii from the Sun represented by particular base sample location $(x, y)$ from Equation 7 . Here we consider that treatment and explore the limits of the approximation used.

The 2-D images from HI1 are integrals through the outer corona and solar wind. To summarize the geometry, we introduce the angle $\xi$, which is the angle between a given feature and the local "sky plane" at a given image location, relative to the Sun itself. This angle is shown in Figure 11. Over a very wide range of $\xi$, called the "Thomson plateau" by Howard \& DeForest (2012), radiance at the focal plane due to a particular density feature is approximately independent of $\xi$. The Thomson plateau causes the line-of-sight integral to extend over a range that is significant compared to the observer-Sun distance $R_{\text {obs }}(\sim 150 \mathrm{Gm})$, mixing both spatial scales and feature-sun distances in the image plane.

Both the scale and radial-offset effects depend on solar elongation angle $\varepsilon$ of a given feature. At low $\varepsilon$, pixels receive significant brightness contributions from a range of line-of-site positions $\Delta s$ that is small compared to $R_{o b s}$, while at higher $\varepsilon$, each pixel receives brightness contributions from a broader $\Delta s$ that is comparable to $R_{o b s}$. So at greater elongations from the Sun, more cross-scale mixing occurs.

The other mixing effect of import is mixing across different values of $r$. The reported brightness in each pixel is a brightness integral along a particular line of sight. The integrand is significant along a finite range of positions $s$ both ahead of and behind the Thomson Surface. This means that most of the sampled wind along a given line of sight is from locations $r(s)$ that are farther from the Sun than $b$ (the impact parameter of the line of sight, i.e., the minimum value of $r$ along the line of sight). We consider and quantify both these effects.

To quantify radial mixing and scale mixing, we identified the range of sky angles $\xi$ that are important to a given pixel in the HI1 images. The Thomson plateau is defined by the geometric $G(\chi)$ effective per-electron scattering function described by Howard \& DeForest (2012). $G(\chi)$ mixes the illumination function and the inefficiency of Thomson scattering at right angles, and is quite flat over the range illustrated in Figure 11. But $G(\chi)$ is useful for comparing radiance of isolated hypothetical features with similar density, and we are concerned instead with a collection of wind features whose collective density falls as $r^{-2}$. Because of this trend in density (Equation 2), the line of sight integral is better described as

$$
B(\varepsilon, \alpha)=k \int n_{e 0} \frac{G(\chi(s, \varepsilon))}{(r(s, \varepsilon))^{2}} d s \approx k\left\langle n_{e 0}\right\rangle(b(\varepsilon))^{-2} \int \frac{(b(\varepsilon))^{2} G(\chi(s, \varepsilon))}{(r(s, \varepsilon))^{2}} d s,
$$

where $B$ is the radiance observed along a given line of sight, $k$ is a scaling factor containing the various constants described by Howard \& DeForest (2012), $n_{e 0}$ is a radially-scaled electron density, the angle brackets indicate line-of-sight averaging, the split factor of $(b(\varepsilon))^{2}$ eliminates the $\varepsilon$ dependence of the integrand (because $\left.r \propto b\right)$, and the integrand at right, which we call $G_{r}(\chi)$, is just the $G(\chi)$ described by Howard \& DeForest (2012), scaled by the known average falloff rate of the solar wind density. $G_{r}(\chi)$ has no plateau and it has a magnitude above 0.5 for only a $78.5^{\circ}$ range of angles, compared to the $114^{\circ}$ range for $G(\chi)$. Taking this range as the effective limit of the line-of-sight integral for each pixel, the spread in spatial scales is approximately the ratio of distances $z$ to the observer at the nearer and farther limits of the angular range. The distance $z$ is just $R \sin (\pi-\varepsilon-\chi) \sin (\chi)^{-1}$. The ratio of scales sampled in a particular small patch of image therefore depends on the elongation $\varepsilon$ of that patch from the Sun. The relationship is plotted in Figure 13. Over the range of elongations $\varepsilon$ considered in this work, the ratio of the largest to smallest scale that affects the structure function at a given apparent separation $\Delta \varepsilon$ or $\Delta \alpha$ reaches a maximum of about 1.8. This has the effect of smoothing out high-polynomial-order variations in the image structure functions, but does not-in first approximation 


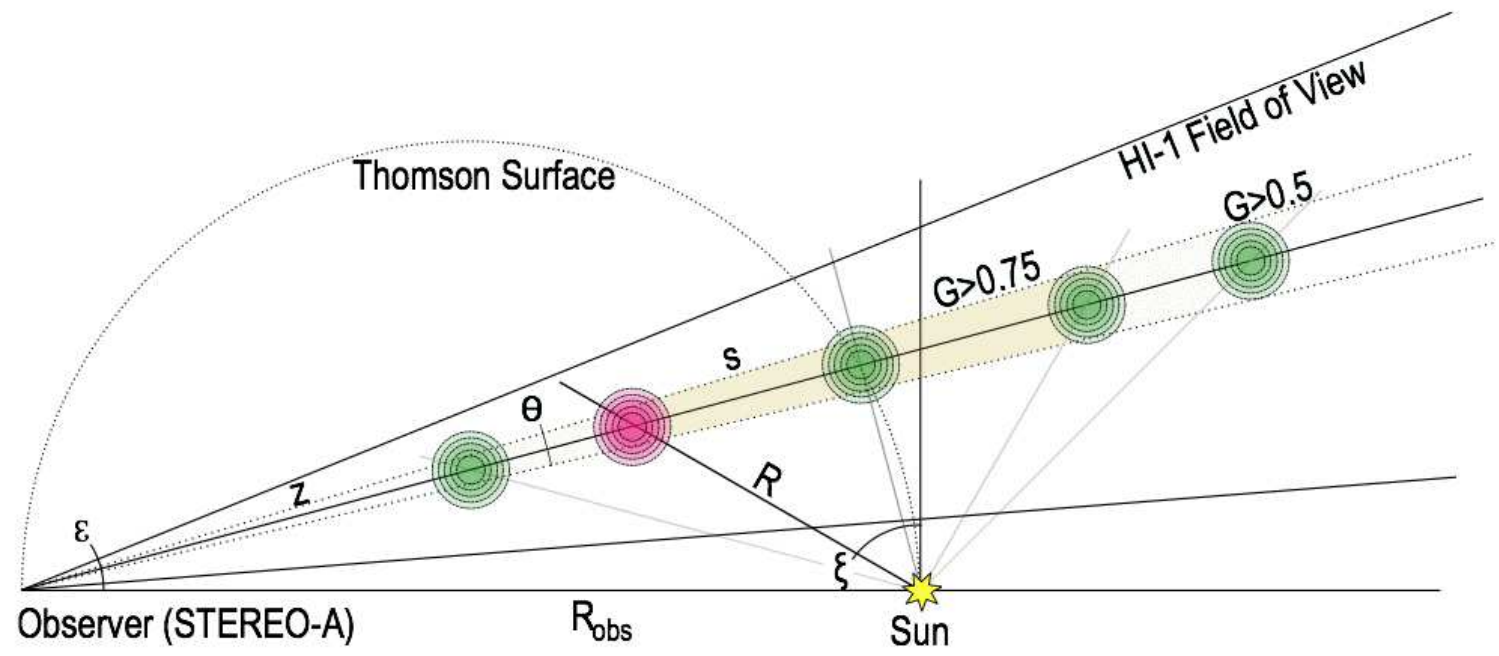

Figure 11. Perspective diagram shows the difficulty of converting from (angular) image scales to spatial scales in the structure function. The geometrical factor $G$ relating density to radiance is nearly flat for a range of approximately $90^{\circ}$ in the out-of-plane angle $\xi$ along a given line of sight. As elongation angle $\varepsilon$ increases, the Thomson plateau grows sufficiently broad that wind structures from many different spatial scales contribute to image features of a given apparent size.

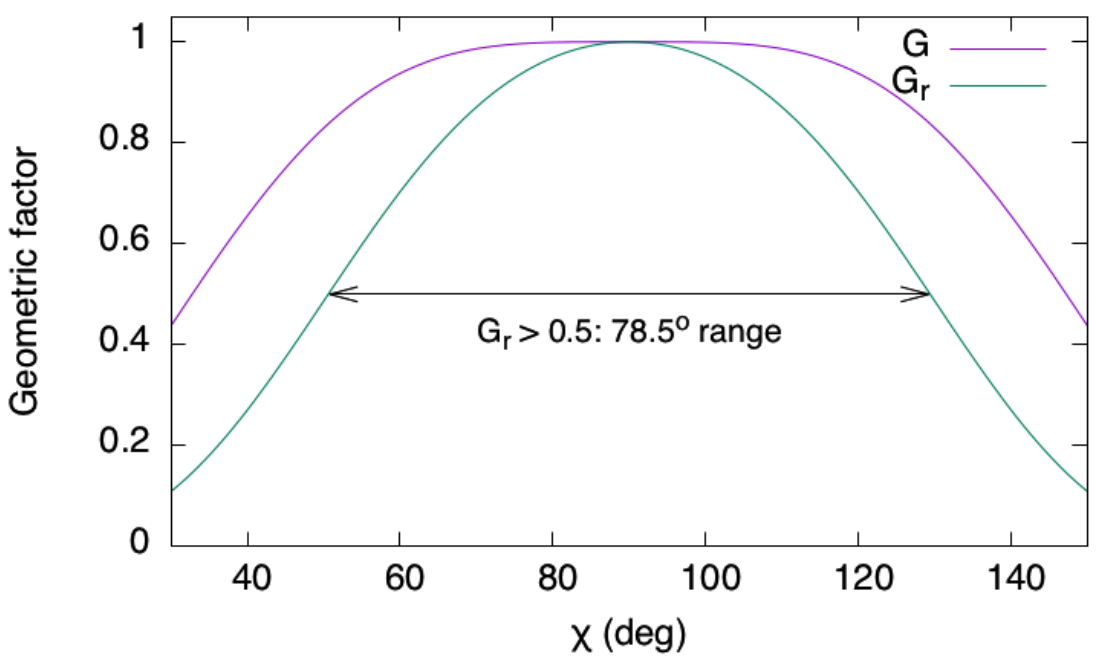

Figure 12. The modified geometric term $G_{r}(\chi)$ is appropriate for determining the effective length of the line-of-sight integral along each pixel of a HI1 image when viewing the distributed background solar wind rather than a single bright isolated feature. The $78.5^{\circ}$ range of angles for which $G_{r}(\chi)>0.5$ is approximately two thirds the $114^{\circ}$ range for which $G(\chi)>0.5$.

order-affect the slope of the nearly-linear sidewalls of the structure function cuts shown in Figures 7 and 8.

Similarly, integrating along the line of sight in Figure 11 yields a weighted average of features sampled at different radii $r$ from the Sun. The most obvious effect is that the average solar distance of a given line of sight is greater than the plane-of-sky distance $b$. We name this average distance $b_{\text {eff }}$ and calculate it as:

$$
b_{e f f}=\frac{\int G_{r}(\chi) \sec \left(\tan ^{-1}(s / b)\right) d s}{\int G_{r}(\chi) d s} \approx \frac{\int_{\chi=50.75^{\circ}}^{\chi=129.25^{\circ}} \sec \left(\tan ^{-1}(s / b)\right) d s}{1.63} \approx 1.09 b
$$

where the approximation treats the integral as a simple average over the FWHM of $G_{r}(\chi)$. So the radial effect results in a lineof-sight $b_{\text {eff }}$ that is just under $10 \%$ higher than the calculated sky-plane distance $b$ itself; and the range of radii sampled along a given line of sight is approximately $\pm 10 \%$ about the expanded $b_{\text {eff }}$.

This $b_{e f f}$ is the line-of-sight impact parameter value that we used in converting angular (image) values to spatial values, for Figure 8 and subsequent discussion. 


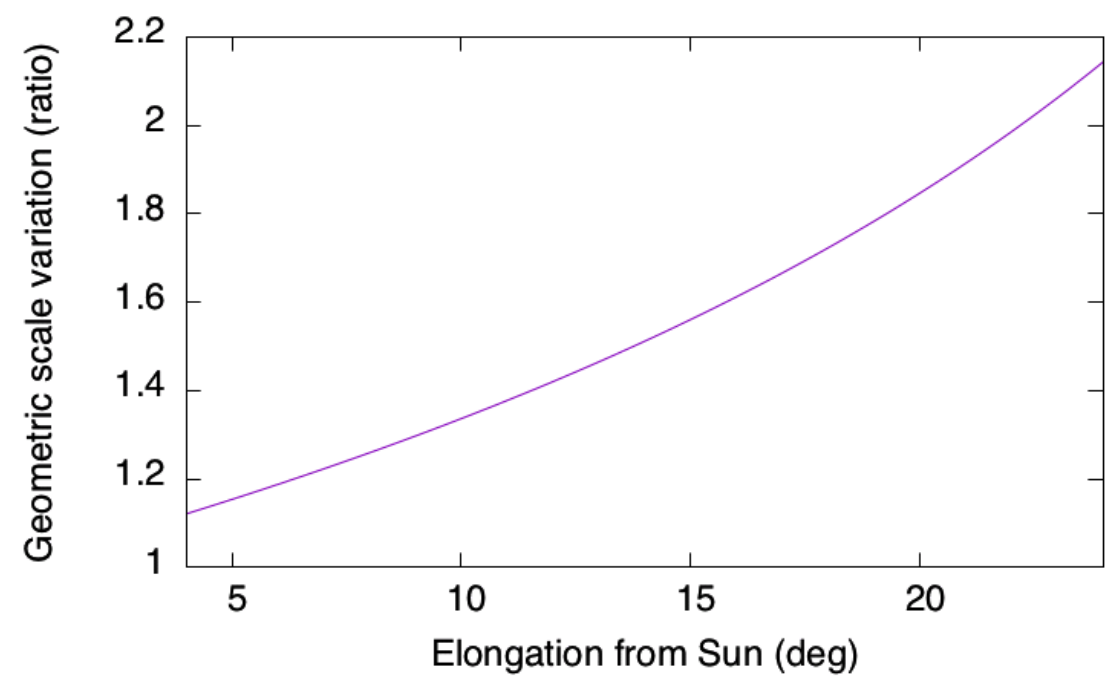

Figure 13. Calculated geometric scale ratio in the image 2-D structure function describes cross-scale smearing against elongation angle $\varepsilon$. The image structure function at $5.7^{\circ}$ (left panels of Figure 7) contains about $\pm 10 \%$ variance in spatial scale, while the structure function at $20^{\circ}$ (right panels of Figure 7) contains about $\pm 40 \%$ variance in spatial scale. 